\title{
Lipid Transporters: From Bacteria, Protozoa, Fungi and Plants, to Mice and Men
}

Satish R. Malwal, ${ }^{1, \#}$ Kyung-Hwa Baek, ${ }^{2, \#}$ Trong-Nhat Phan, ${ }^{2}$ Hyeryon Lee, ${ }^{2}$ Zhu-Hong Li ${ }^{3}$, Silvia N.J. Moreno ${ }^{3}$, Joo Hwan $\mathrm{No}^{2,}$, and Eric Oldfield ${ }^{1, ~ *}$

${ }^{1}$ Department of Chemistry, University of Illinois at Urbana-Champaign, Urbana, IL 61801, USA

${ }^{2}$ Leishmania Research Laboratory, Institut Pasteur Korea, Seongnam-si, Republic of Korea

${ }^{3}$ Center for Tropical and Emerging Global Diseases and Department of Cellular Biology, University of Georgia, Athens, GA 30602, USA

* Corresponding author

E-mail: eoldfiel@illinois.edu; joohwan.no@ip-korea.org

\# These authors contributed equally

\section{Keywords}

SQ109, MmpL3, Niemann-Pick, Cholesterol, GIPCs, Transporters, Tuberculosis 


\begin{abstract}
The tuberculosis drug candidate SQ109 targets the trehalose monomycolate transporter MmpL3 in Mycobacterium tuberculosis and also has activity against other pathogens. We found related proteins in 22 protozoa, including Trypanosoma cruzi and Entamoeba histolytica, as well as in archaea and other bacteria, including the fatty acid transporter, FarE. We show these proteins, $\alpha$ MMPL proteins, adopt similar structures to that of MsMmpL3 having two sets (P1 and P2) of conserved active site/ $\mathrm{H}^{+}$-transporter Asp, Tyr and Phe residues in "pentad" motifs (DYxxF) that can bind to the SQ109 ethylenediamine and adamantyl moieties. Based on structural comparisons with MsMmpL3, we find that there are superimposable transmembrane and $\mathrm{H}^{+}$-transporter structures in much larger proteins, $\beta$-MMPLs, found in apicomplexan parasites, fungi, plants and animals. They also contain double "pentad" motifs in which the P1 Asp is totally conserved, but the P2 Asp may also be a Glu, and the P2 Phe seen in the $\alpha$-MMPLs is a His that H-bonds to the P1 and P2 Asp/Glu residues. There are also 5 conserved Ser/Thr residues that extend the Hbond $/ \mathrm{H}^{+}$-transporter network with 2 interacting directly with the $\mathrm{P} 1 \mathrm{Asp}$, and the His. We propose that all MMPL proteins are involved in proton motive force-mediated lipid (phospholipid, glycolipid, sterol, fatty acid) transport, and that SQ109 may target some pathogens directly, by binding to the P1/P2 motifs. Overall, the results are of general interest since they indicate that there are two major classes of lipid transporters: $\alpha$-MMPL proteins found in many bacteria and protozoa, and much larger, $\beta$-MMPL proteins, found in fungi, apicomplexa, plants and animals and, in some cases, they are potential drug targets.
\end{abstract}




\section{Introduction}

One path to the discovery of new drugs or drug-leads is to repurpose an old drug, used for a different disease. For example, in the case of visceral leishmaniasis, amphotericin B (used to treat fungal infections) and miltefosine (originally developed as an anti-cancer drug) have been successfully repurposed ${ }^{1}$. Another example is ivermectin, originally developed to treat heartworm in dogs, that is now used extensively to treat onchocerciasis (river blindness ${ }^{2}$ ). In our work and in work with our collaborators, we reported that the anti-tubercular drug candidate ${ }^{3,4}$ SQ109 [Nadamantan-2-yl-N'-((E)-3,7-dimethyl-octa-2,6-dienyl)-ethane-1,2-diamine], 1 in Figure 1, has potent activity against kinetoplastid parasites in vitro ${ }^{5-8}$. In $M$. tuberculosis, the compound interferes with cell-wall assembly by inhibiting the essential enzyme MmpL3, Mycobacterium membrane protein Large 3, a trehalose monomycolate (TMM, 2) transporter ${ }^{9}$, and a crystal structure of SQ109 bound to MsMmpL3 has been reported ${ }^{10}$. SQ109 is also active against the fungi Candida albicans and Aspergillus fumigatus ${ }^{11}$ as well as the apicomplexan parasite Plasmodium falciparum $^{12}$.

The wide range of activity of SQ109 raises the question as to whether there might be "MmpL3like" (MMPL) proteins in these and other organisms and here, we use a combination of sequence, structural as well as structure-prediction information, as outlined schematically in Figure $1 \mathrm{~b}$, to probe for the presence of MMPL proteins in archaea, bacteria, protozoa, fungi, plants as well as in animals, together with an in vivo investigation of the activity of SQ109 against T. cruzi, T. brucei, L. donovani and Toxoplasma gondii infected mice. The results were unexpected in that we find that there appear to be two very similar types of MMPL proteins, both involved in lipid transport: small ones that are involved in e.g. TMM, fatty acid and phospholipid binding/transport and hereinafter called $\alpha$-MMPLs, and much larger proteins ( $\beta$-MMPLs) such as the Niemann-Pick 
C1 (NPC1) or Niemann-Pick C1-like (NPC1L1) cholesterol (CHOL, 3$)^{13}$ transporters, found in animals, in addition to structurally- related transporters found in yeasts, ${ }^{14}$ apicomplexan parasites ${ }^{15}$ as well as in plants ${ }^{16}$ that appear to be involved in phospholipid—primarily sphingolipid (SL, there are many, one is shown in Figure 1a as 4) — transport. Both the $\alpha$-MMPLs and the $\beta$ MMPLs have a similar arrangement of 12-13 transmembrane (TM) helices as well as "active site" (as opposed to substrate-binding) residues involved in $\mathrm{H}^{+}$-transport in the TM domain, while the large extra-membranous regions, known to be involved in CHOL/ERGO uptake/trafficking or at least binding in yeasts and animals, are only found in the $\beta$-MMPL proteins. Notably, it has recently been discovered ${ }^{17}$ that the antifungal azoles itraconazole $\mathbf{5}$ and posaconazole $\mathbf{6}$ potently inhibit the Niemann-Pick C1-like protein and a structure has been reported, results of interest here since we reported previously ${ }^{5}$ that SQ109 acts synergistically with posaconazole in $T$. cruzi. In other work, ${ }^{18}$ it has been found that, remarkably, the glycolipid trehalose dimycolate (TDM) as well as TMM formed by M. tuberculosis can inhibit NPC1 resulting in a NPC-disease phenotype in infected macrophages that contributes to Mtb persistence, so the NPC1 protein can bind both the sterol CHOL as well as a glycolipid (and as discussed more below, fatty acids), leading to the general idea that both $\alpha$-MMPLs as well as $\beta$-MMPLs are rather promiscuous as to which lipids they transport, or at least, bind.

Here, we investigated potential targets for SQ109 in bacteria and protozoa finding that both $\alpha$ and $\beta$-MMPLs were quite widespread, leading to more general proposals for the structures and functions of these proteins in which not only are the TM structures similar, but so are the $\mathrm{H}^{+}-$ translocating domains. In particular, we explore the concept that both $\alpha$ - and $\beta$-MMPLs contain superimposable dual "pentad" motifs that are involved in catalyzing proton-motive-force driven transport. In the $\alpha$-MMPLs the motifs are both DYxxF and the molecules that are transported are 
e.g. TMM and fatty acids (and others, phospholipids, bind), ${ }^{19}$ while in the $\beta$-MMPLs the motifs are generally DDxx(V/L/I) in $\mathrm{P} 1$ and $(\mathrm{D} / \mathrm{E}) \mathrm{xxxH}$ in $\mathrm{P} 2$ and the molecules that are transported can be sterols such as cholesterol, phospholipids and even a fatty acid. We explore to what extent the pentad motifs, containing the polar residues that are essential for catalysis and are involved in $\mathrm{H}$ bonding $/ \mathrm{H}^{+}$-transport, align between the $\alpha$ - and $\beta$-MMPLs, and report the discovery of five highly conserved Ser/The residues in the $\beta$-MMPLs systems that are proposed to be part of the $\mathrm{H}^{+}$ transporter network. And finally, we report the first in vivo results for SQ109 against four pathogenic protozoa. 

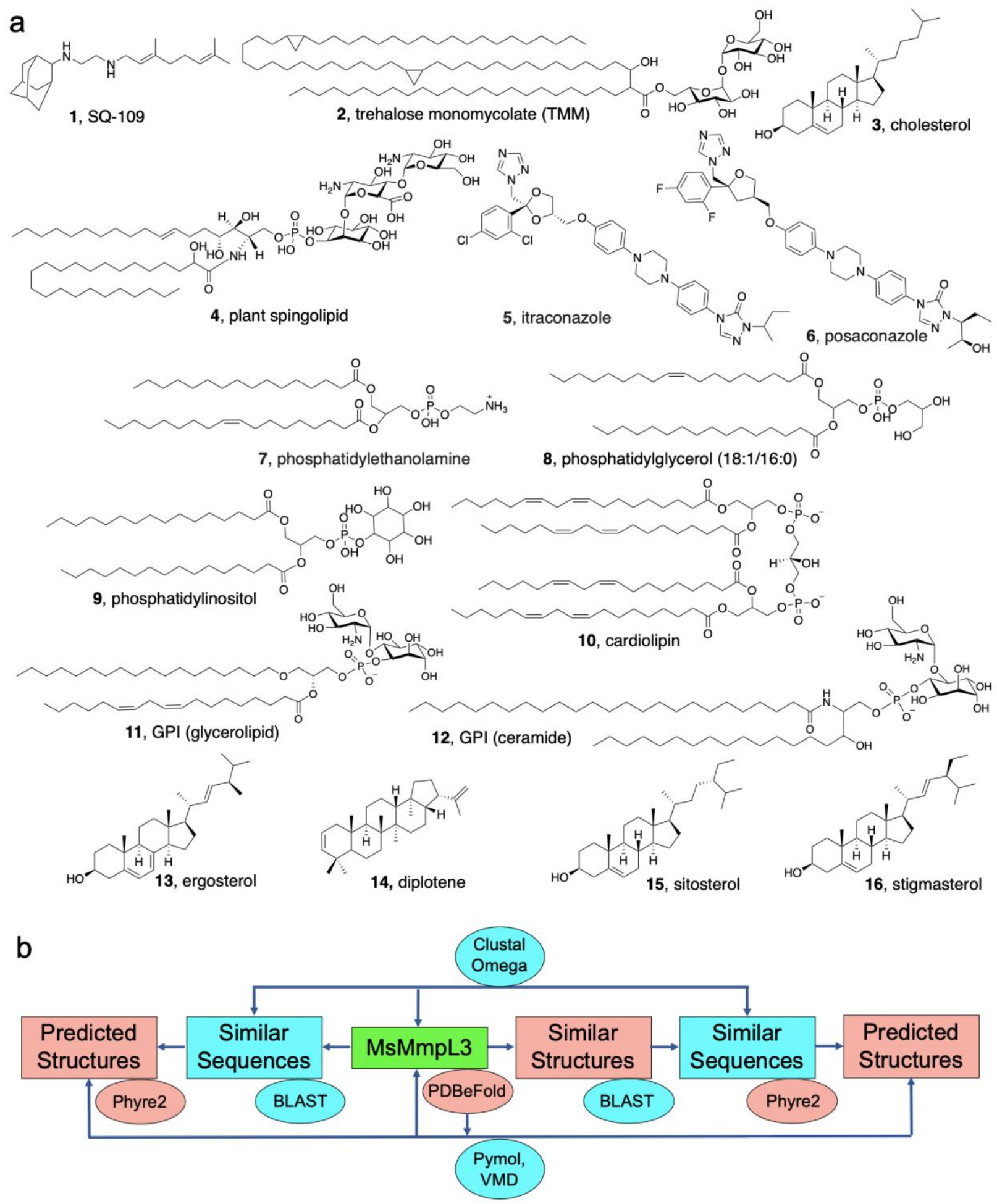

Figure 1. Structures of compounds discussed in the Text and a flow-chart for finding MMPL proteins. a) Structures of compounds. b) Flow-chart showing routes to identification of $\alpha$ and $\beta$ - 
MMPL sequences and structures using BLAST, PDBeFold/SSM, Phyre2, Clustal Omega and Pymol/VMD computer programs. MsMmpL3 (green) is the Mycobacterium smegmatis TMM transporter MmpL3 and serves as the query for both sequence as well as structure-based searches.

\section{Results and Discussion}

Discovery of MmpL3-like proteins in Trypanosoma cruzi and in other protozoa. When considering the possible mechanisms of action of SQ109 it seemed likely that there might be an MmpL3-like protein in one of more of the parasitic protozoa investigated here, since that could help explain parasite growth inhibition
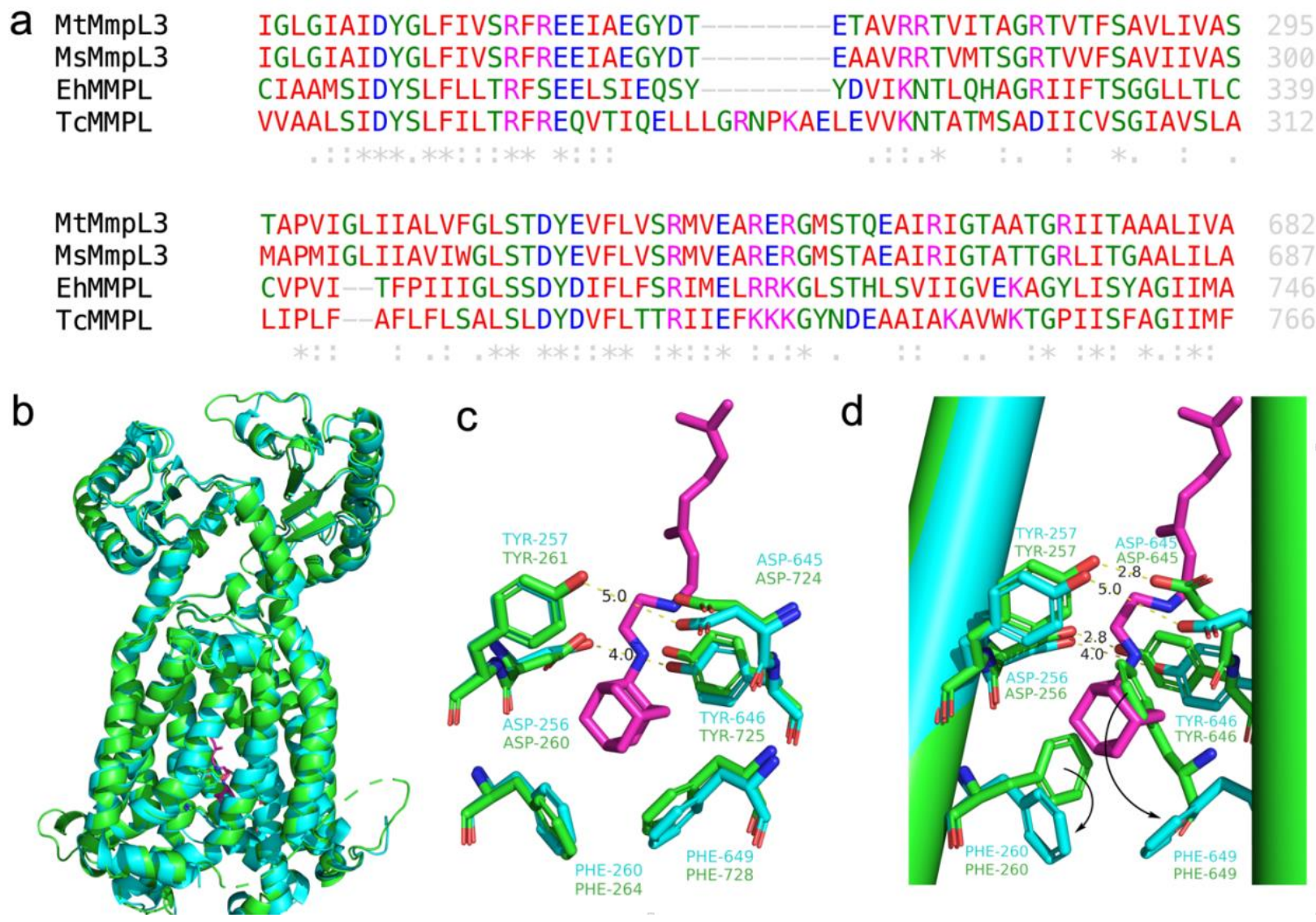
Figure 2. Proposed structure of the T. cruzi MmpL3-like protein (TcMMPL), not found in other trypanosomatid or apicomplexan parasites, and sequence alignment results. a) Sequence alignment of MsMmpL3, M. tuberculosis MmpL3 (MtMmpL3), TcMMPL and the Entamoeba histolytica MmpL3-like protein (EhMMPL). Full sequence alignments are shown in the Supporting Information, Figure S2. b) Phyre $2^{20}$ structure prediction for TcMMPL (green) superimposed on MsMmpL3 (cyan, PDB ID code $6 \mathrm{AJG}^{10}$ ). SQ109 is shown as pink sticks. c) Key Asp residues that are involved in binding to the SQ109 ethylenediamine moiety in the MsMmpL3 x-ray structure, together with the neighboring Tyr and Phe residues that are involved in hydrophobic interactions with the SQ109 adamantyl group, superimposed on the TcMMPL Phyre2 model. The model is of interest since all 6 residues (2 Asps, 2 Tyrs and 2 Phes) are very closely aligned and suggest why T. cruzi is inhibited by SQ109. d) Alignment of MsMmPL3 bound to SQ109 (cyan, PDB ID code 6AJG) with that of apo-MsMmPL3 (green, PDB ID code 6AJF) showing hydrogen bond distances between active site residues in the presence and absence of SQ109.

by SQ109. To test this hypothesis, we carried out a BLAST ${ }^{21}$ (a sequence-based) search of all eukaryotes using M. tuberculosis MmpL3 as a query, as shown in Figure 1b. Sequence results are shown in Supporting Information Table S1 and Figure S1. The results show that essentially all proteins that have similar sequences to the M. tuberculosis MmpL3 (MtMmpL3) are found in protozoa, and these include two human pathogens: T. cruzi, the causative agent of Chagas disease, and Entamoeba histolytica, the causative agent of amebic dysentery. Notably, there were no similar sequences in the other trypanosomatids or in any apicomplexan parasites, or indeed in fungi or humans - at least as revealed by the $\mathrm{BLAST}^{21}$ sequence-based search, although there was one hit in Arabidopsis thaliana, but not in any other plant. A partial sequence alignment of the T. cruzi 
protein (here called TcMMPL), the E. histolytica protein (EhMMPL), MtMmpL3 as well as the M. smegmatis protein (MsMmpL3), whose structure has been reported ${ }^{10}$, is shown in Figure 2a. A full sequence alignment of these four proteins is shown in Figure S2. There are two sets of Asp, Tyr and Phe residues (DYxxF) that interact with the SQ109 ethylenediamine and adamantyl moieties seen in the x-ray structure of SQ109 bound to MsMmpL3, and these are also present in the T. cruzi and E. histolytica protein, Figure 2a. We propose to call these motifs the first and second sets of "pentad" residues, P1 and P2, since this facilitates a discussion of similar sets of residues in other organisms. Due to the arrangement of amino-acid side-chains in an $\alpha$-helix, the first, second and fifth residues are relatively close and in many cases are conserved and are involved in catalysis.

Next, we used the Phyre2 program ${ }^{20}$ to predict the structure of the TcMMPL and EhMMPL proteins, both of which we classify as $\alpha$-MMPLs. The full predicted structure of TcMMPL is shown in Figure 2b, superimposed on that of the MsMmpL3/SQ109 complex (PDB ID code 6AJG), and a zoomed-in version is shown in Figure 2c. As can be seen in Figure 2c, SQ109 is likely to bind to TcMMPL just as it does in the mycobacterial protein. The same results were obtained with EhMMPL (data not shown). It thus appears that in T. cruzi there is an MMPL protein that is likely to be targeted by SQ109. Interestingly, using again a sequence-based approach, we also found $\alpha$-MMPL proteins in two archaea, Euryarchaeota archaeon and Candidatus Poseidoniales archaeon, in addition to finding similar predicted structures (not shown).

The result shown in Figure 2c is of interest in the context of how SQ109 might bind, however, in the absence of an inhibitor, as described elsewhere ${ }^{12}$, there are major conformational changes in this "active site" region that are important for making comparisons with other $\alpha$ - as well as $\beta$ MMPL proteins. In particular, in the absence of an inhibitor, Figure 2d, there are clearly hydrogen 
bonds between the Asp and Tyr residues in each DYxxF motif with heavy atom distances of $\sim 2.8$ $\AA$ and these distances increase to $\sim 4-5 \AA$ in the presence of SQ109 (and most other inhibitors). In the absence of SQ109, the 2 Phe groups bind in an "up" position but are moved "down", Figure $2 \mathrm{~d}$, in the presence of most inhibitors, plus, there are obvious changes in the orientation of one TM helix. The importance of the movement of the Phe groups is that in the other, larger, MMPL proteins ( $\beta$-MMPLs), one of the conserved Phes seen here is replaced by another aromatic residue, a His, that is essential for activity and forms part of an alternative H-bond network, containing conserved D/E residues, and this His binds in the "up" position.

MMPL domains are present in animal proteins. The observation of $\alpha$-MMPL proteins in $T$. cruzi and E. histolytica was unexpected since these organisms do not utilize trehalose monomycolate, a very large, hydrophobic molecule and indeed, in very early work the TcMMPL was not detected, due we believe to our use of an early and incomplete $T$. cruzi genome data base. However, we found no BLAST hits in other eukaryotes, as expected based on conventional wisdom that e.g. a MtMmpL3-like protein is absent in humans. It was thus surprising to see that when we carried out a structure-based search using the PDBeFOLD/SSM (secondary structure matching) server ${ }^{22}$ with MsMmpL3 as the query and against all $~ 170,000$ reported PDB structures, there were in fact several proteins that contained very similar 3D structures to that of the bacterial, MsMmpL3 protein. Three of these were in animals and were patched, dispatched, and NiemannPick $\mathrm{C} 1$ protein (NPC1), another was the hopanoid transporter $\mathrm{HpnN}$ found in Burkholderia spp. as well as in many other hopanoid-producing species. Patched and dispatched are involved in hedgehog signaling in insects and vertebrates and NPC1 is involved in cholesterol trafficking and a review of their general structures and ligand transport mechanisms has recently been reported ${ }^{13}$ 
- though not in the essential, active site/ $\mathrm{H}^{+}$-transporter regions of interest here.

NPC1-like proteins are also found in yeasts, such as Candida albicans and Aspergillus fumigatus - SQ109 targets—as well as in the apicomplexan parasites $P$. falciparum and $T$. gondii that are also inhibited by SQ109—although any similarity in the active site/ $\mathrm{H}^{+}$-transporter regions is only apparent after using the initial structure-based NPC1 "hit" as a BLAST sequence query, due to overall low sequence identity between MsMmpL3 and the NPC1-like proteins. These proteins we generically term $\beta$-MMPL proteins.

In patched, dispatched and NPC1, all $\beta$-MMPLs, we find that there is a $\sim 3 \AA \mathrm{C} \alpha$ rmsd over $\sim 500$ residues when compared with the structure of the $\alpha$-MMPL, MsMmpL3. Figure S3a shows a superposition of the MsMmpL3 structure (PDB ID code 6AJF) with that of human patched (PDB ID code $6 \mathrm{OEU}$ ) in which the $\mathrm{C} \alpha$ rmsd is $2.67 \AA$ /511 residues; Figure S3b shows a superposition of MsMmpL3 with that of Drosophila melanogaster dispatched (PDB ID code 6TBU) in which the $\mathrm{C} \alpha$ rmsd is $2.76 \AA$ /494 residues, and Figure S3c shows a superposition with Niemann-Pick C1 (PDB ID code 5U74) in which the C $\alpha$ rmsd is $3.45 \AA / 567$ residues. As noted above, these structural similarities were not detected in the initial BLAST search (using MsMmpL3), which is based solely on amino acid sequences, because the overall sequence homology is low. There is also structural similarity between MsMmpL3 and the Burkholderia multivorans hopanoid transporter $\mathrm{HpnN}$ (PDB ID code 5KHN) ${ }^{23}$ and that of MsMmpL3 (6AJ5), as shown in Supporting Information Figures S4a and S4b, and with Saccharomyces cerevisiae NPC1 (PDB ID code 6R4L $\mathrm{L}^{21}$ ), an apparent sphingolipid (phospholipid) transporter (C $\alpha$ rmsd is $3.32 \AA / 543$ residues). Zoomed-in views of the structures of MsMmpL3/SQ109 with the animal proteins in the SQ109 ligand binding region in MsMmpL3 are shown in Figures S3d-f where it is apparent that there is a Phe to His change in the animal proteins. This region is involved in $\mathrm{H}^{+}$-driven sterol transport in 
the Niemann-Pick proteins ${ }^{24}$ and is also found in the Saccharomyces cerevisiae NPC system (NCR1 and NPC2) and is discussed more extensively below. But how similar are the structures and sequences of the proteins discussed above to other MMPL domain-containing proteins?

A common active site structure in all MMPL proteins? Given the observation that a structurebased search using MsMmpL3 as a query resulted in the discovery of several much larger proteins involved in sterol and phospholipid trafficking, we next used several of these NPC1 and NPC1like proteins as BLAST queries against the apicomplexans $P$. falciparum and $T$. gondii as well as the fungi $C$. albicans and A. fumigatus - since these organisms are all inhibited by SQ109 and the observation of similarity to MsMmpL3 means that there must be an $\alpha$-MMPL domain in these larger, $\beta$-MMPL proteins. We find that each of these four organisms contains at least one $\beta$-MMPL protein that resembles e.g. human NPC1 (HsNPC1), but no smaller $\alpha$-MMPL proteins, such as found in MsMmpL3. P. falciparum and T. gondii do not synthesize sterols, but take up cholesterol (CHOL) from host cells ${ }^{25,26}$, while $C$. albicans and A. fumigatus both produce ERGO as their main membrane sterol. However, in $T$. gondii as well as in another fungus $S$. cerevisiae, it appears that (unesterified) sterol transport is not the primary function of these proteins, rather, it is sphingolipid transport $^{7,8}$. That notwithstanding, given the presence of NPC1 or related proteins in each of these four species, it seemed possible that each might be one target for SQ109 and that if that were the case, they should all have very similar local structures that enable SQ109 to bind, as it does in MsMmpL3. They could also not be targets, but the question still remains as to what might their structures be? In particular, are there $\mathrm{H}^{+}$-pump or sensor motifs that are similar to those found in the $\alpha$-MMPL protein MsMmpL3? 
To investigate this in more detail we carried out a Clustal Omega ${ }^{27}$ sequence alignment of 18 proteins that encompasses all of the bacterial, protozoal, fungal and animal systems discussed above in addition to an NCR1 protein, found in many plants, 
A DmDispatched HsDispatchedH1I1 EaMMPL

CdFarE

SaFarE

CPaMMPL

MtMmpL3

MsMmpL3

EhMMPL

TCMMPL

MmPatched

EhNPC1

AfNCR1

CaNCR1

AtNCR1

HsNPC1

PfNCR1

TgPatched

b

DmDispatched HsDispatchedH1I1

EaMMPL

CdFarE

SaFarE

CPaMMPL

MtMmpL3

MsMmpL3

EhMMPL

TCMMPL

MmPatched

EhNPC1

AfNCR1

CaNCR1

AtNCR1

HSNPC1

PfNCR1

TgPatched
GADDVFLFLKIWHCVLTERFSNRCTLTTOSOSALPTLENSDHTESLENIMALTMRHAAAS GADDAFVLCDVWNYTKFDKPH___ AETSETVSITLQHAALS GTDYSIFLVSRYRDERRDGRT___ SIDYALFIVSRYRAELLRGGA $-\mathrm{P}-$ SDAIGRAVGTAGSA GIDYALFILFRYRQVMKTETD $\quad Y \quad-$ IKGIGLAIGTAGSA SVDYSLFIVNRFREELGRGHD _- R - RTAIAVTSATAGRA AIDYGLFIVSRFREEIAEGYD-_- $-\mathrm{T}-$ ETAVRRTVITAGRT AIDYGLFIVSRFREEIAEGYD-_- $-\mathrm{T}-$ EAAVRRTVMTSGRT SIDYSLFLLTRFSEELSIEQS - $-Y \quad Y$ YDVIKNTLOHAGRI

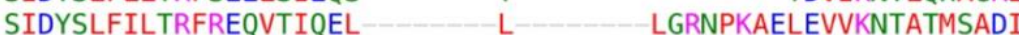
GVDDVFLLAHAFSETG - QN - KRIPFEDRTGECLKRTGAS GVDNIFILTNTIDEQPMYDKDG_-_-OYVPVEKRLEHSLMHVGPS GVDNIFLIVHEFERIN - VSH-_ PDEEIDERVARAVGRIGPS GIDNIFLIVHELHVIS - EGN- PNLALEVRISQALKHIGPS GVDNMCILVHAVKRQE-_-_- ELPLERRISNALMEVGPS GVDNIFILVQAYQRDE - RL- - QGETLDQQLGRVLGEVAPS GVDDVFVILNSYSLLFMVK- DNKKRIQMCLKDSALA GVDDMFVVMNCYCVSYTIH___ DPEERCIQALRISGLG

VAVLILL-GWQLN- ILESIAVSTAIGLAVDFSLHYGIHYRMSPVKE VGSLVLL-GWELN- - VLESVTISVAVGLSVDFAVHYGVAYRLAPDPD LALTILLFHVWRGID - VIFILPLALFVMAMGLGMDYDIFIIT - RVREEVA FGVTVAIFQEGWLGL - ITHTQPIISFLPIFLIGVVFGLAMDYQVFLVT - RMREEFM LGFTTLVMQDGFMKG LFGIETTGPMLAFLPVITIGILFGLAMDYEVFLMS - RIHEEYS FGVLVWVFQEGNGSE-LLNF-TPQPIDPTTPVMIFAILFGLSMDYEVLMLS- RIHEEWE MGILTWIFVDGHFSK -WLNF-TPTPLTAPVIGLIIALVFGLSTDYEVFLVS- RMVEARE MGILTWMFVDGHGSG - LMNY-TPQPLMAPMIGLIIAVIWGLSTDYEVFLVS --RMVEARE YGFASFVFCNDYFDWTSQIMKESIGLYWCVPVITFPIIIGLSSDYDIFLFS - RIMELRR FGVGVVVFQYNWLHPVWKALDGVGNFCFLIPLFAFLFLSALSLDYDVFLTT - RIIEFKK FGMMGLI GIKLS AVPVILIASVGIGVEFTVHVALAFLTAI IGIMYLW - GVELN- AVSCVNLVMSIGITIEFCVHIAHAFLSSPK IGSMAIA GVSLN AVSLVNLVICVGIGVEFCAHIARAFMFPSRTI GGVLALW - SISLN_—AVTLVNLVICVGFAVEFTIHLTRAYCVPKVKM LGVMAVF-HIQLN ALSVVNLIMSVGIAVEFCVHITHAFSISTFGVMWLW-GISLN-AVSLVNLVMSCGISVEFCSHITRAFTVSMK FGFMCLC - GITMN $\quad$ IISMVILVLSVGFSIDHTSHIVQAFSHSMG FGFMYFW - RVKLH- MVSTIALVISIGFAVDYSAHLCHTFTHCKG

C

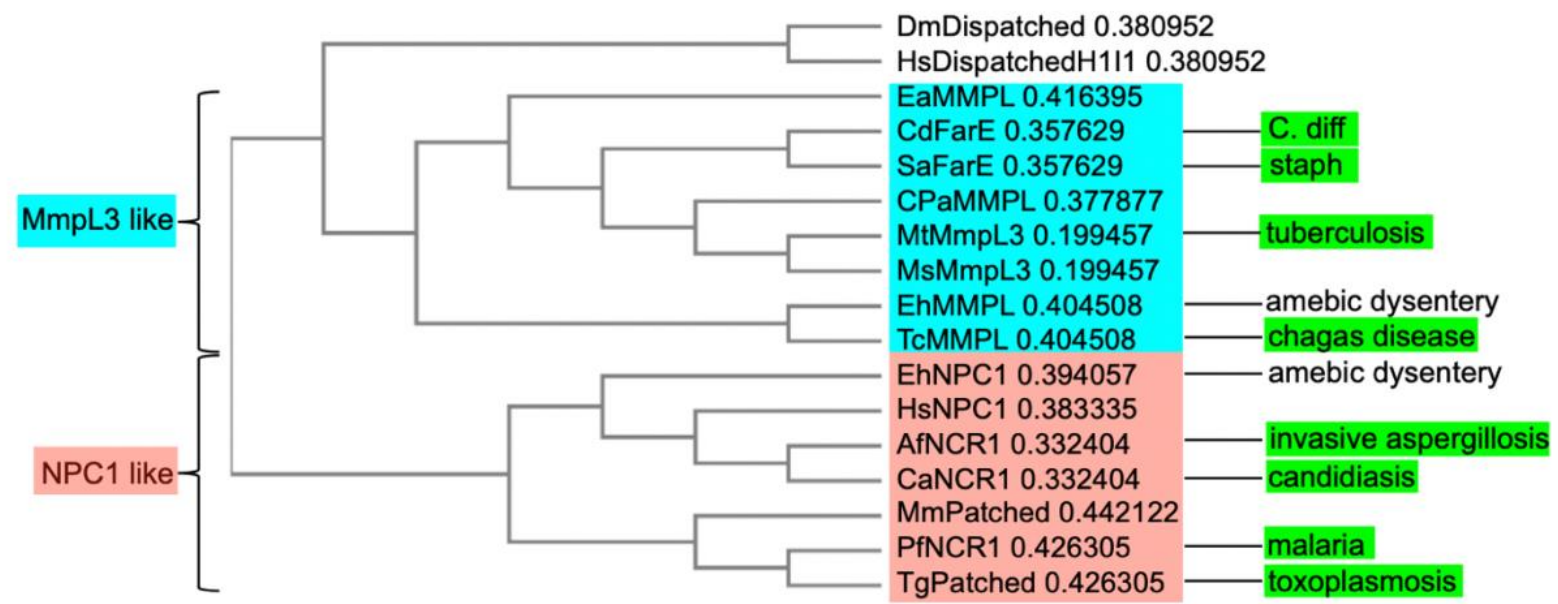

Figure 3. Partial Clustal Omega ${ }^{27}$ alignments of MMPL proteins from bacteria, archaea,

protozoa and animals. $\mathrm{Cd}=$ Clostridium difficile; $\mathrm{Ea}=$ Euryarchaeota archaeon $; \mathrm{CP}=$ Candidatus

Poseidoniales archaeon; $\mathrm{Sa}=$ Staphylococcus aureus; $\mathrm{Mt}=$ M. tuberculosis; $\mathrm{Ms}=$ M. smegmatis; 
$\mathrm{Eh}=E$. histolytica $; \mathrm{Dm}=$ Drosophila melanogaster $; \mathrm{Hs}=H$. sapiens $; \mathrm{Tc}=T$. cruzi $; \mathrm{Af}=A$. fumigatus $;$ $\mathrm{Ca}=C$. albicans $; \mathrm{Mm}=$ Mus musculus $; \mathrm{Pf}=P$. falciparum $; \mathrm{Tg}=T$. gondii. Other abbreviations are given in the Text.

derived from a BLAST search using human NPC1; the S. aureus fatty acid transporter FarE and its homolog found in C. difficile, CdFarE, and two archaea (from a BLAST search of archaea using MsMmpL3 as a query). This gives a reasonably comprehensive set of results for archaea, bacteria, protozoa including apicomplexans, fungi, a plant, and animals. More comprehensive results with plants are discussed below. Sequence alignment results are shown in Figures $3 \mathrm{a}$ and $3 \mathrm{~b}$ and a phylogram is shown in Figure 3c.

Of the 18 sequences investigated there are on average about 1000 residues per protein, but only one is totally conserved: the Asp in the first DYxxF-like pentad motif (P1) in MsMmpL3, Figure 3a, that in MsMpL3 is involved in $\mathrm{H}^{+}$transport and is close to the SQ109 ethylene diamine group in the MsMmpL3 structure. Both DYxxF pentads (P1 and P2) are very highly conserved in the mycobacteria, the archaea as well as in the T. cruzi and E. histolytica proteins, and in the fatty acid transporters such as Staphylococcus aureus $\operatorname{FarE}^{28}$ (SaFarE), while the pentads are different in apicomplexans, fungi, plants and animals. More specifically they are generally $\mathrm{D}(\mathrm{D} / \mathrm{N}) \mathrm{xxx}$ in $\mathrm{P} 1$ and (D/E) $x \times x H$ in P2. The major difference between the $\alpha-$ and $\beta$-MMPLs is thus the loss of the $\mathrm{Y}$ in P1 and P2 and addition of a P1 Asp and a P2 His, in the $\beta$-MMPLs. This appears to be of interest since there is a puzzle as to why in the case of NPC1 and NPC1-like proteins, protondriven transport results in cholesterol import, while in the bacterial transporter MsMmpL3 (or MtMmpL3), there is TMM export, as with fatty acid export in FarE. Both processes are driven by 
the PMF and the main difference from a structural or electrostatic perspective is that there is a potentially large difference in the arrangement of charged residues in the "active site" that might result in a different response to the PMF. Specifically, in both the $\alpha$ - and $\beta$-MMPLs proteins there are always two anionic residues, Asp or Glu, having side-chain pKa values of $~ 4$. In MsMmpL3, the two Asps form part of a hydrogen bond network, interacting with the two Tyrs, thought to be part of the "proton-wire". However, in the larger $\beta$-MMPL proteins the Tyr groups are generally absent and the P2 Phe in MsMmpL3 is now a totally conserved P2 His. Plus, there is generally a second Asp in the P1 motif. Since His contains an imidazole side-chain which is quite basic having pKa values of $\sim 6$, there is expected to be a very different charge distribution to that in e.g. MsMmpL3 and if the imidazole is protonated, there will be a large dipole moment with the Asp/Glu residues, that will interact with the PMF and potentially, influence the direction of flux of a ligand of interest. We discuss in the next section the pentad sites in the small and large MMPLs in more detail. Remarkably, in very early work on the $\beta$-MMPL NPC1, Davies et al. ${ }^{29}$ expressed NPC1 in Escherichia coli finding that NPC1-containing cells accumulated both acriflavine as well as oleic acid with a 15-fold increase in oleic acid uptake upon IPTG induction, noting that "the polarity of NPC1 is reversed in relation to that of E. coli AcrB, even though these proteins share the same membrane topology". That is, NPC1 and related proteins can act as importers (of CHOL, sphingolipids, oleic acid) and not as exporters (of e.g fatty acids as in SaFarE, or TMM in $\alpha$ MMPLs), and the main obvious difference in the "active site" region where protonation is thought to occur is the introduction of the conserved His, a basic residue, in addition to a set of 5 conserved Ser/Thr residues that extend the H-bond network in the $\beta$-MMPLs, as we describe below.

The $\mathbf{H}^{+}$-sensor "active sites" in $\alpha$ - and $\beta$-MMPL proteins. In recent work it was hypothesized that there might be a role for $\mathrm{Na}^{+}$rather than $\mathrm{H}^{+}$in energizing $\mathrm{CHOL}$ transport by patched ${ }^{30}$, an 
NPC1-related protein, since there are typically no large $\mathrm{pH}$ gradients in mammalian cell plasma membranes. However, in later work ${ }^{31}$ the opposite effects were observed, that is the $\mathrm{K}^{+}$gradient and not the $\mathrm{Na}^{+}$gradient was proposed to control sterol uptake. A model for $\beta$-MMPL activity in which the two Asps and a Glu seen in the "active site" in patched (that are also present in the apicomplexan NPC1-like proteins) or the three Asps in dispatched, was proposed in which a cation effects a conformational change by involving hydrogen-bond interactions with one or two Thr/Ser residues. However, upon inspection of the sequences and structures of dispatched, patched and the yeast SL transporter, as well as of the apicomplexan and plant sequences, Figures $3 a, b$, it can be seen that the second Asp in P1 in the $\beta$-MMPL proteins is often an Asn, which would not bind $\mathrm{Na}^{+}$. Rather, as noted above, it is the His that is totally conserved and this His is essential for activity in the yeast system, as shown by mutagenesis experiments ${ }^{24}$. In addition, the protonophore uncoupler CCCP ( $m$-chlorophenyl carbonylcyanide phenylhydrazone) has been reported to decrease the activity of patched, expressed in a yeast system ${ }^{32}$. As shown in Figures 3 and S3, it thus appears that a major difference between the $\alpha$ - and $\beta$-MMPLs is that in former, both sets of Asps and Tyr are highly conserved and in most cases $\mathrm{P} 2$ is a DY(D/E)xF, the third D/E being part of the "proton wire"19. In the $\beta$-MMPLs (e.g. NPC1, patched) there is now a His in place of the Phe seen in the smaller proteins and this would be expected to take part in a proton relay and when protonated, would generate a large dipole moment (somewhat tilted from the bilayer normal), due to the presence of the anionic Asp and Glu residues, and the interaction of this dipole with $\Delta \psi($ a non-uniform electric field) due to a cation gradient could contribute to a conformational change. In any case, these structural suggestions necessitated a more detailed analysis of the P1 and $\mathrm{P} 2$ domains in the $\alpha$ - and $\beta$-MMPLs, and later, of their environs. 
Rotation/translation analysis of P1 and P2 motifs in $\alpha$ - and $\beta$-MMPLs. In order to try and better understand the nature of the "active sites" in the $\alpha$ - and $\beta$-MMPLs, we investigated the structures of the $\mathrm{P} 1$ and $\mathrm{P} 2$ motifs (in the $\mathrm{H}^{+}$-binding active site region) in $\beta$-MMPL proteins then made a comparison with MsMmpL3+SQ109. Figure 4a shows an alignment of HsNPC1 with ScNCR1. Figure $4 b$ shows the predicted "active site" in a PfNCR1 Phyre2 model. Figure 4c shows a comparison of the PfNCR1 model with MsMmpL3+SQ109. Then, we carried out a multistructure comparison using the PDBeFold/SSM server ${ }^{22}$ of the structures of MsMmpL3, HsNPC1, and HsPatched together with the Phyre2-predicted structures of TcMMPL, SaFarE, PfNCR1 and a TgNCR1. 

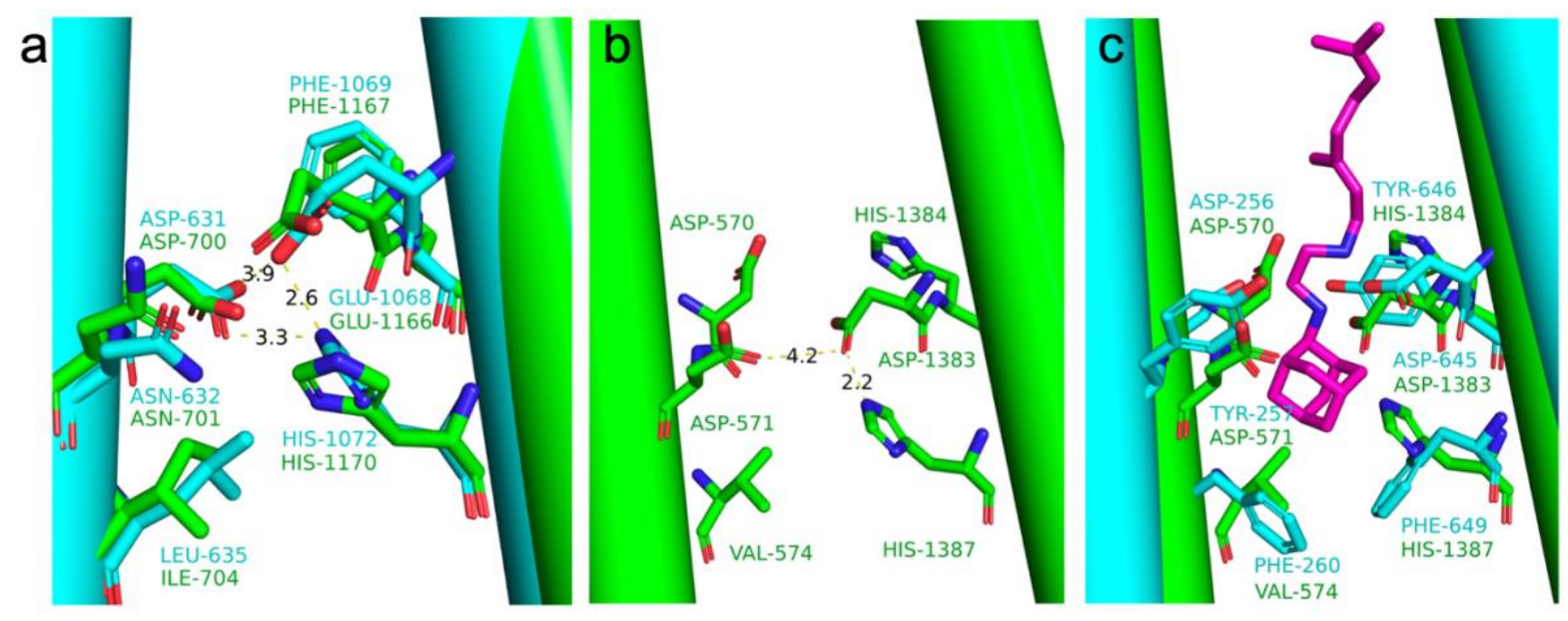

\begin{tabular}{|c|c|c|c|c|c|c|}
\hline MsMmpL3 & TcMMPL & SaFarE & HsNPC1 & HsPatched & PfNCR1 & TgNCR1 \\
\hline ASP 256 & ASP 260 & ASP 245 & ASP 700 & ASP 513 & ASP 570 & ASP 463 \\
\hline TYR 257 & TYR 261 & TYR 246 & ASN 701 & ASP 514 & ASP 571 & ASP 464 \\
\hline GLY 258 & SER 262 & ALA 247 & ILE 702 & VAL 515 & VAL 572 & MET 465 \\
\hline LEU 259 & LEU 263 & LEU 248 & PHE 703 & PHE 516 & PHE 573 & PHE 466 \\
\hline PHE 260 & PHE 264 & PHE 249 & ILE 704 & LEU 517 & VAL 574 & VAL 467 \\
\hline ASP 645 & ASP 724 & ASP 603 & GLU1166 & GLU1095 & ASP1383 & ASP1008 \\
\hline TYR 646 & TYR 725 & TYR 604 & PHE1167 & PHE1096 & HIS1384 & TYR1009 \\
\hline GLU 647 & ASP 726 & GLU 605 & CYS1168 & THR1097 & THR1385 & SER1010 \\
\hline VAL 648 & VAL 727 & VAL 606 & SER1169 & VAL1098 & SER1386 & ALA1011 \\
\hline PHE 649 & PHE 728 & PHE 607 & HIS1170 & HIS1099 & HIS1387 & HIS10121 \\
\hline
\end{tabular}

Figure 4. Structures of the first $\mathrm{DDxF}(\mathrm{V} / \mathrm{L} / \mathrm{I})$ and second $(\mathrm{D} / \mathrm{E}) \mathrm{xxxH}$ motifs in the $\mathrm{H}^{+}$-binding active site region in (most) large MMPL proteins and a comparison with MsMmpL3+SQ109. a) HsNPC1 (green, PDB ID code 5U74) with ScNCR1 (cyan, PDB ID code 6R4L). b) Active site in PfNCR1, Phyre2 model. c) PfNCR1 model (green) with MsMmpL3+SQ109 (cyan, PBD ID code 6AJG). d) PDBeFold/SSM multi-protein alignment of apo-MsMmpL3, TcMMPL model, SaFarE model, HsNPC1, HsPatched (6OEU), PfNCR1, and TgNCR1.

As can be seen in Figure 4a, there is a very similar H-bond network in HsNPC1 and ScNCR1 that involves the highly conserved Asp and Glu residues and the totally conserved His with HisGlu and His-Asp distances in the $2.6-4 \AA$ A range. The same pattern is seen in the PfNCR1 model, 
Figure 4b, where remarkably, there are three Asps and two His in the malaria parasite protein, a strong candidate for a $\mathrm{H}^{+}$sensor. The PfNCR1 model is shown superimposed on the structure of MsMmpL3+SQ109 in Figure 4c and suggests a SQ109 binding site, with the His moving down as do the Phes in MsMmpL3 on the binding of most inhibitors. That is, the His is "up" because there is no inhibitor present. When the 3D structures ( $\mathrm{x}$-ray, cryo-EM and predicted) of the seven proteins: MsMmpL3, TcMMPL, SaFarE, HsNPC1, HsPatched, PfNCR1 and a TgNCR1 proteins are all compared using the PDBeFold/SSM program, which involves translation/rotation of all structures to obtain a minimum $\mathrm{C} \alpha$ root mean squared deviation (rmsd), Figure $4 \mathrm{~d}$, it is apparent that the P1 Asp is totally conserved and that the main differences between the $\alpha$-MMPL proteins (like MsMmpL3) and the $\beta$-MMPLs — of course in the $\mathrm{H}^{+}$-transporter region-is that e.g. MmpL3 and the fatty acid transporters have a pair of Asp-Tyrs while the larger proteins have a network of Asp, Glu and His residues, expected to be sensitive to $\mathrm{pH}$ or PMF effects, due to their dipolar nature, basically as discussed briefly above.

All of the acidic residues (Asp, Glu) are shaded in cyan in Figure 4d, the Tyr are orange while the His are green. In the $\alpha$-MMPLs, the Phe that are in both P1 and P2 are shaded pink. In the $\beta$ MMPLs, the P1 Phes are now the aliphatics Val, Leu or Ileu, but are in essentially the same spatial location as the Phes in MsMmpL3, TcMMPL and SaFarE, while the P2 Phes seen in the $\alpha$ MMPLs are now all His residues that can H-bond to, primarily, the Asp or Glu residues in P1 and P2. There are, therefore, clear structural similarities between the $\alpha$ - and $\beta$-MMPLs but also, what appears to be a major difference, the replacement of a Phe by a His, essential for activity. It is also of interest to note that when comparing the structures (one x-ray, one predicted) of the TMM transporter MsMmpL3 with that of the known fatty acid transporter SaFarE, the only difference between the 10 pairs of residues that comprise P1 and P2 is that there is a Gly>Ala change-and 
these residues are actually outside the P1 P2 "active site" region. This indicates that the $\mathrm{H}^{+}-$ transporter region is highly conserved in proteins that have very different functions, although as we noted above, an MMPL protein may be able to carry out the transport of diverse molecules, as with the CHOL/fatty acid transport activity of HsNPC1. In addition, there is another major difference between the $\alpha$ - and $\beta$-MMPLs in that there is now a network of Ser/Thr in the $\beta$ MMPLs.

Role of Ser and Thr residues in the active site. In previous work ${ }^{31}$ it was proposed that there were two Asps in e.g., HsPatched that interacted with a cation $\left(\mathrm{Na}^{+}\right)$and that one of these Asps (the second one in the P1 DDxF(V/L/I)x motif) interacted with a Thr. Moreover, it was shown that the Thr was involved in catalytic activity. How then does this observation relate to the "Asp/Glu/His" H-bond network interaction model? To investigate this to see if there might be a role for the conserved Thr in our "model", we inspected the structure alignment for the 7 proteins (3 $\alpha$-MMPLs and $4 \beta$-MMPLs) discussed above (determined by use of the PDBefold/SSM server), searching for highly conserved Ser/Thr-rich regions. Results for Ser and Thr residues in the vicinity of the catalytic Asp, Glu and His residues in the $\beta$-MMPL proteins (HsNPC1, HsPatched, PfNCR1 and TgNCR1 models) are shown in Figure 5a (in yellow), together with results for MsMmpL3, TcMMPL and SaFarE.

As can be seen in Figure 5a, there are typically $5 \mathrm{Ser} / \mathrm{Thr}$ residues that are highly conserved in the $\beta$-MMPL proteins and there are no other similar "clusters" in the 7-protein alignment. We show in Figure 5b structural, that is H-bond network distance results, for HsPatched, for comparison with previous work. Based on the cryo-EM structure of HsPatched (PDB ID code 6OEU) and using the reported residue numbering, it appears that there is a "proton-wire": Ser-1132 > Thr- 
$1133>$ Asp-513 > Glu-1095 > His-1099 with 4 heavy atom hydrogen bond distances of $3.2 \pm 0.50$ $\AA$. So, there is actually good accord with the observation that the Thr, Asp, Glu and His are all essential for catalytic activity in the $\beta$-MMPLs ${ }^{31,12}$. In addition, we see that there is a second set of highly conserved Ser/Thr residues, in HsPatched: Thr-551, Ser-552, and Ser-554, Figure 5a. As shown in Figure $5 \mathrm{~b}$, Thr-551 is $\sim 3.2 \AA$ from the His, indicating the presence of a H-bond, while Ser-552 and 554 are more distant (7-9 А) —-though they could be involved in H-bonding during the PMF-driven conformational change during transport since they are so highly conserved.

These sequence and structural results are of importance because they complement the more obvious interactions with (potentially) charged residues by providing in essence, an "outer sphere" set of (neutral) Ser/Thr that can stabilize the more obvious Asp/Tyr/Glu/His interactions seen (or proposed here) in the $\beta$-MMPL proteins. It is also of note that the first residue is the "NPC-like" Ser/Thr alignment (Ser-738 in NPC1; Thr-551 in HsPatched; Thr-606 in PfNCR1 and Thr-499 in TgNCR1) is also present in the $\alpha$-MMPLs MsMmpL3 (as Ser-293) and TcMMPL (Ser-305) and in the case of MsMmpL3, Ser-293 was previously proposed to be part of the "proton-wire"19.

Things may be somewhat more complex in that Ser-293 only interacts with Asp-256 and Tyr645 in the SQ109-liganded form of MsMsmpL3 - although this structure might well reflect what happens, structurally, during catalysis. That notwithstanding, the observation of Ser or Thr in this position in 6/7 structures (or proposed structures) investigated does suggest a key role in catalysis, involving $\mathrm{H}^{+}$-transport. In HsPatched, this residue would be Thr-551, which is H-bonded to His1099, although it should be noted that the protonation, tautomer or ring-flip isomer state of the His is not known. Taken together, the results described here indicate that there are two H-bond networks in the $\beta$-MMPL proteins (like HsPatched) that stabilize the conserved Asp, Glu and His residues essential for catalysis. It also seems less likely that the second Asp (in the P1domain) is 
directly involved in binding to a metal cation, since in some proteins it is the neutral Asn, but it is still part of a H-bond network. The arrangement of potentially charged residues as well as of Ser/Thr H-bond partners (in the two clusters) is shown in cartoon form in Figure $5 \mathrm{c}$ in which Ser/The are shown as yellow spheres, Asp/Glu are cyan spheres and His are green spheres, so based on the available evidence it appears that there are generally 8 residues that are conserved in this region and these are likely to represent the $\mathrm{H}^{+}$-sensor.

\begin{tabular}{ccccccc}
\hline MsMmPL3 & TcMMPL & SaFarE & HsNPC1 & HsPatched & PfNCR1 & TgNCR1 \\
\hline SER 293 & SER 305 & ALA 282 & SER 738 & THR 551 & THR 606 & THR 499 \\
\hline ALA 294 & GLY 306 & GLY 283 & SER 739 & SER 552 & THR 607 & THR 500 \\
VAL 295 & LEU 307 & VAL 284 & PHE 740 & ILE 553 & LEU 608 & LEU 501 \\
ILE 296 & ALA 308 & THR 285 & SER 741 & SER 554 & THR 609 & THR 502 \\
& & & & & & \\
ILE 685 & ILE 764 & ILE 643 & LEU 1204 & SER 1132 & SER1421 & SER 1046 \\
LEU 686 & MET 765 & MET 644 & THR 1205 & THR 1133 & THR1422 & THR 1047 \\
\hline
\end{tabular}

b
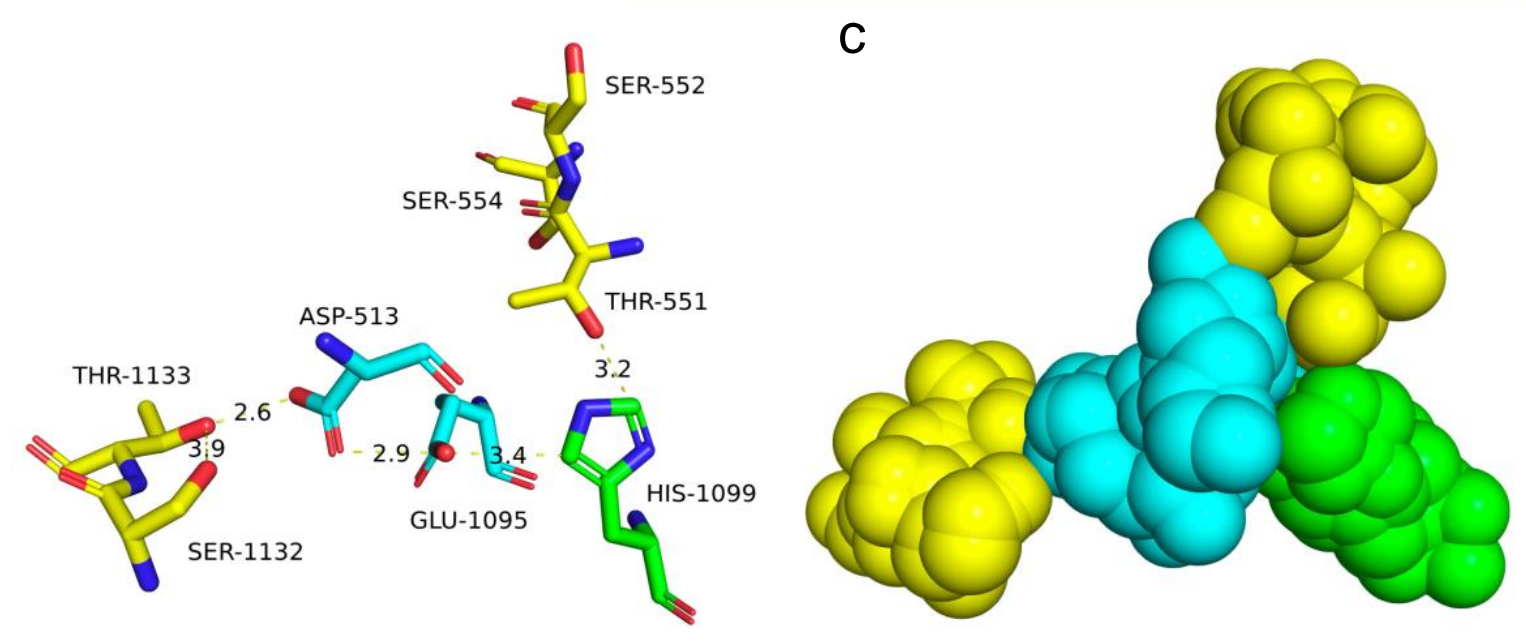

Figure 5. Conserved Ser/Thr clusters in $\beta$-MMPL proteins. a) Ser/Thr residues (in yellow) from a PDBeFold/SSM alignment of the 7 proteins indicated. MsMmpL3, HsNPC1 and HsPatched are from experimental structures while the others are from computational models. b) H-bond networks in HsPatched showing Ser/Thr residues (yellow), Asp/Glu (cyan) and His (green), from PDB ID 
code 6 OEU. The distances shown are in $\AA$ units. The protonation/tautomer/ring-flip status of the His is not known. c) Space filling view of a superposition of HsNPC1, HsPatched, PfNCR1 and TgNCR1 showing conserved Ser/Thr (yellow), Asp/Glu (cyan) and His (green).

Lipid transport in MMPL proteins. Next, we consider possible transporter roles for the trypanosomatid and apicomplexan MMPL proteins. In very recent work $^{33}$, the structure of a complex between the Niemann-Pick proteins C1 and C2 (PDB ID code 6W5V) has been reported in which three cholesterol (CHOL) molecules were identified. This structure is of particular interest since as shown in Figure 6a, when it is compared with that of MsMmpL3 containing a lipid ligand, phosphatidylethanolamine (PE, 7), it is apparent that one of the three CHOL (yellow) and PE (pink) molecules can occupy the same binding site in both proteins, Figure 6a. While the NPC1/NPC2 molecules are larger than is MsMmpL3, the same folds and one lipid binding site are present in the TM regions in both systems. But what lipids might be transported by the TcMMPL protein whose sequence and structure we discussed above?

In MsMmpL3 the protein transports trehalose monomycolate 2, with trehalose being a disaccharide. However, it has also been reported that the phospholipid phosphatidylethanolamine 7 binds, as do several other phospholipids—phosphatidylglycerol 8, phosphatidylinositol (PI, 9) and cardiolipin 10, suggesting that in $T$. cruzi, TcMMPL might act as a lipid transporter. In $T$. cruzi, one of the three largest families of proteins in the genome ${ }^{34}$ is involved in the synthesis of mucins, highly glycosylated phospholipids that have a so-called GPI, glycosylphosphatidylinositol $(11,12)$ membrane-anchor ${ }^{35}$. In the early steps involved in mucin biosynthesis, GPIs need to be transported from the cytosol to the endoplasmic reticulum for extensive glycosylation ${ }^{36}$ but the transporter has not been reported and TcMMPL is a likely candidate since the major difference 
between the PI that binds to MsMmpL3 and the GPI anchors in T. cruzi is just the addition of a second sugar residue — and MsMmpL3 of course transports TMM, another lipid-disaccharide. It is also of interest to note that there are a very large number of GPI lipids found in $T$. cruzi and these comprise a very broad range of structures with glycerolipid $\mathbf{1 1}$ or ceramide $\mathbf{1 2}$ cores, diacylation and alkylacylation, and a wide range of alkyl chain-lengths. While these GPIs might all be transported by different proteins, the fact that MsMmpL3 transports another lipiddisaccharide, TMM, and also binds diverse phospholipids, suggests that TcMMPL may be able to transport many different GPIs, in much the same way that a drug efflux pump can efflux more than one drug. In E. histolytica, there are similar mucin-like related GPI-anchored proteins that are involved in pathogenesis ${ }^{37,38}$, suggesting a similar role for EhMMPL.

The second possibility is that TcMMPL (and EhMMPL) might be involved in sterol transport. This may seem implausible — two different molecules transported by the same protein — but as we noted above, in early work on NPC1, Davies et al. ${ }^{29}$ showed that this CHOL transporter, when expressed in E. coli, in fact acted as a fatty acid importer, of oleic acid (and of acriflavine), indicating some promiscuity. In E. histolytica, ergosterol (ERGO, 13) is not synthesized and instead, cholesterol (CHOL, 3) is imported by the EhNPC1/2 $\operatorname{transporter}^{39}$, while in $T$. cruzi, the other pathogenic protozoan with an $\alpha$-MMPL protein, there is again CHOL import ${ }^{40}$ (into reservosomes), but there is no NPC1 to carry out that process. The MmpL3-like proteins might thus (also) be involved in CHOL or ERGO transport, in T. cruzi. This hypothesis is based on the observation that the structures of the proteins MsMmpL3 (or the predicted TcMMPL) and the hopanoid transporter $(\mathrm{HpnN})$ are similar (Figure S4), as are the structures of hopanoids such as diplotene (14, Figure 1) and CHOL. We also reported previously ${ }^{5}$ that SQ109 acted synergistically with posaconazole (an azole anti-fungal drug that targets P450 demethylase, leading to inhibition 
of ERGO biosynthesis), with an FICI (fractional inhibitory concentration index) of 0.48 , indicating a synergistic effect. Since there are often synergistic effects with inhibitors that target the same pathway ${ }^{41}$, inhibition of ERGO transport (via TcMMPL inhibition) would help explain the synergistic activity of SQ109 with posaconazole (it inhibits ERGO biosynthesis), as would our observation that abnormal ergosterol analogs are produced under SQ109 pressure ${ }^{5}$. However, there is also another attractive reason for the synergistic interaction between SQ109 and posaconazole since it has recently been shown that itraconazole, as well as posaconazole, inhibit NPC1, which would be expected to decrease sterol trafficking in host cells, reducing CHOL availability to $T$. cruzi.

In the apicomplexans $P$. falciparum and $T$. gondii, there is no CHOL or ERGO produced by the parasite and CHOL is taken up from host cells in an as yet unspecified manner. There are, however, NPC1-like proteins in both organisms (called PfNCR1 in Plasmodium falciparum; TgNCR1 in T. gondii) and it is possible that these are involved in CHOL uptake and that this process may (or may not, see below) be inhibited by SQ109. Plus, SQ109 might also inhibit host cell NPC1 proteins. As noted above, it has recently been found that itraconazole binds to NPC1 and inhibits its function and since itraconazole inhibits the growth of P. falciparum as well as T. gondii ${ }^{42,43}$, where CHOL is taken up from the host cells, then targeting of either parasite or of host CHOL transport, might be expected. In the malaria parasite P. falciparum, it has been found that under SQ109 drug pressure there is a $\sim 2-3 \mathrm{x}$ increase in the $\mathrm{IC}_{50}$ for cell growth inhibition and that this correlates with the observation of mutations in the vacuolar ATPase ${ }^{44}$. While there is no published evidence that SQ109 directly targets the vacuolar ATPase, these workers also reported a $1.35 \mathrm{x}$ increase in the $\mathrm{IC}_{50}$ in a PfNC1 knockdown, so inhibiting the PMF (driven by ATPase inhibition), 
PfNCR1 as well as red cell HsNC1L1 may all contribute to $P$. falciparum cell growth inhibition, by SQ109.

Finally, we very briefly consider the ligand binding sites in reported $\alpha$ - and $\beta$-MMPL proteins since this leads to a "canonical" picture of the ligand-binding domains. As noted above and as shown in Figure 6a, SQ109 (blue spheres) binds to the $\mathrm{H}^{+}$-transporter region of MsMmpL3 and is below the position of one CHOL in NPC1/NPC2 (yellow, middle of Figure 6a) while PE 7 (in MsMmpL3, shown in pink) binds above that CHOL. The two other CHOLs are shown at the top of Figure 6a. In their paper on the ITRA/NPC1 structure, Long et al. ${ }^{17}$ 


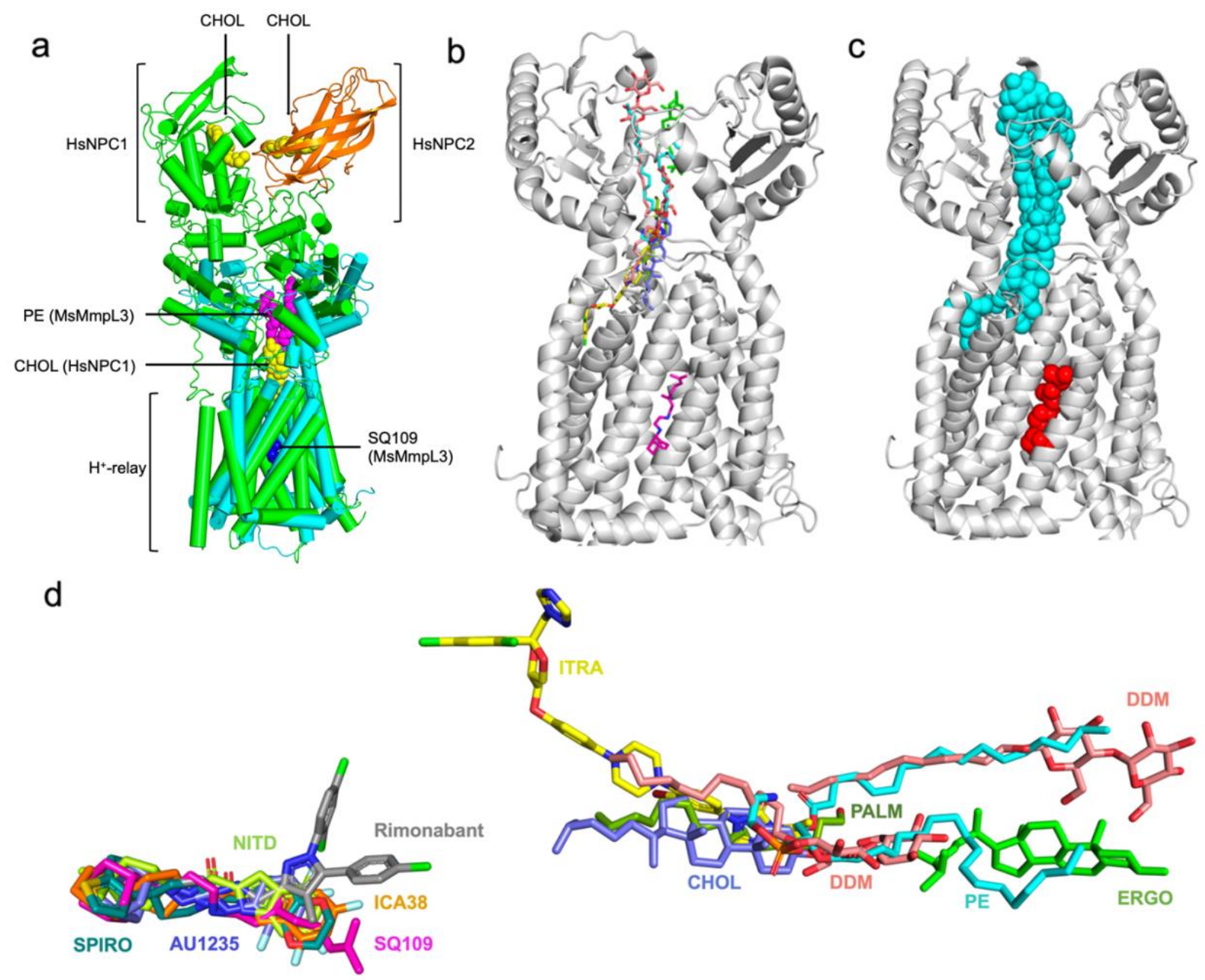

Figure 6. Comparisons between MMPL structures and ligand binding sites. a) MsMmpL3 (cyan, PDB ID code 6OR2) with a bound PE (phosphatidylcholine, pink spheres) superimposed on the structure of NPC1/NPC2 (green/orange cylinders) with three bound cholesterols (yellow spheres, PDB ID code 6W5V). Also shown is the position of SQ109 (blue spheres) in the MsMmpL3 structure (PDB ID code 6AJG). b) Structure of MsMmpL3 bound to SQ109 (PDB ID code 6AJG) superimposed on aligned ligands from other MMPL structures: MsMmpL3 bound to phosphatidylethanolamine (PDB ID code 6OR2); human PTCH1 bound to palmitate (PDB ID code 6E1H); human NPC1 bound to itraconazole (PDB ID code 6UOX); NPC1-NPC2 complex structure bound to CHOL (PDB ID code 6W5V); and S. cerevisiae Niemann-Pick type C protein 
NPC1 bound to ERGO (PDB ID code 6R4L). Ligands are shown as sticks. c) Same as b) showing “tunnel binding" ligands as cyan spheres, SQ109 as pink spheres. d) Zoomed-in view of the ligands shown in b) together with other MmpL3 inhibitors, and their names. The view is rotated $90^{\circ}$ from that shown in b).

reported the structure of human NPC1 with ITRA bound and noted that there was a tunnel into which CHOL, ERGO as well as ITRA could bind. This tunnel is similar to that predicted computationally in the earlier work ${ }^{23}$ on $\mathrm{HpnN}$, although there, only the cavity and not its occupant (as determined by $\mathrm{x}$-ray crystallography) was reported. We show in Figure $6 \mathrm{~b}$ the MsMmpL3 protein (grey cartoon) together with the positions of various ligands: SQ109, ITRA, CHOL, ERGO, PE and PALM (the palmitate moiety in a hedgehog structure), together with two dodecyl maltoside (DDM, detergent) ligands from the MsMmpL3/SQ109 structure. Figure 6c shows ligands in the tunnel (cyan spheres) together with SQ109 (red spheres) and Figure 6d shows all the ligands that bind to MsMmpL3 $3^{10}$, labelled for clarity. Clearly, there is a tunnel (cyan) that can transport (or bind) a wide variety of ligands including CHOL and ERGO, TMM, PE as well as, we propose, phospholipids such as the GPI anchors involved in mucin biosynthesis in T. cruzi and E. histolytica, and fatty acids (as in SaFarE, CdFarE). With the fatty acid transporters, the observation of a palmitate ligand (PALM) in the 2:1 human PTCH1-Shh-N complex (PDB ID code $6 \mathrm{E} 1 \mathrm{H})^{45}$ suggests that fatty acids may bind to this site also. The conformational changes that effect transport are driven by the PMF that is decreased by SQ109, but not by all MmpL3 inhibitors, and these as well as SQ109 all bind to the central TM region (red in Figure 6c).

The idea that "MmpL3" like proteins are absent in humans, pathogenic yeasts and fungi, Trypanosoma cruzi and Plasmodium falciparum is incorrect, and it appears that there are very 
similar lipid and potentially, inhibitor binding sites in several of the pathogens. Does that mean that SQ109 might be toxic, if it inhibits cholesterol transporters? Fortunately, there have not been any reports of SQ109 toxicity in Phase 2b/3 clinical trials and there is in fact a cholesterol lowering drug, ezetimibe, that binds to HsNPC1L1 ${ }^{46}$.

Structure and $\beta$-MMPL active sites in plants. Another question of interest relates to plants. Many plants contain sterols such as sitosterol (15) and stigmasterol (16) and as noted some time ago, the plant $A$. thaliana contains two proteins, NCR1 and NPC2, that have sequence similarities to e.g. human NPC1 and NPC2, with NPC2 being a very small protein originally designated (in fungi) as a phosphatidylglycerol/phosphatidylinositol transfer protein. ${ }^{47}$ The question is then, do most plants have the same fundamental heterodimer structure (i.e. NCR1/NPC2), or do some function more like the monomeric proteins seen in e.g. T. gondii? To explore these questions, we carried out BLAST searches of all plants using the S. cerevisiae NCR1 and NPC2 sequences (whose protein structures are known). There were 1032 NCR1 hits returned, and 499 for NPC2. This suggested a $~ 2: 1$ NCR1/NPC2 composition, due perhaps to the presence of both NCR1/NPC2 heterodimers with other, $\beta$-MMPL monomers. However, on closer inspection we found that there were 299 "isoforms" in the NCR1 data set but only 29 in the NPC2 data set, plus, there were many NCR1 strain differences (but not NPC2 strain differences), leading to the conclusion that most plants contain a $\sim 1: 1$ NCR1-NPC2 heterodimer used, based on the results with A. thaliana, in sphingolipid trafficking. The BLAST results with the $\beta$-MMPL proteins show similar conserved P1 and P2 motifs to those discussed above with highly conserved Asp, Glu and His residues and show quite convincingly that plants contain the same "active site" $\mathrm{H}^{+}$-transporter motifs as seen in the fungal, apicomplexan and animal proteins. A comparison between the HsNPC1/NPC2 
structure and the predicted structure of a plant heterodimer (from Quercus suber) is shown in Figure S5a and the Ser/Asp/Glu/Thr H-bond network is shown in Figure S5b, together with a longer-range interaction with the His.

It should be noted here that there are some ambiguities in the literature with respect to the NPC2 proteins. First, NPC2 in the plant literature in some cases refers to a phosphatase that has $\sim 514$ amino-acids, $\sim 3 \mathrm{x}$ larger than the NPC2 in S. cerevisiae and in plants. Second, the S. cerevisiae NPC2 whose structure has been reported is annotated (in the PDB, the Protein Data Bank) as well as in NCBI BLAST search results, as a phosphatidylglycerol $(\mathbf{8}$, PG)/phosphatidylinositol $(\mathbf{9}$, PI) transferase. This is because in early work ${ }^{47}$ a fungal NPC2 protein from A. oryzae having strong sequence homology to the S. cerevisiae protein (that can bind ergosterol) was found to transfer PG and PI between membranes-both model and biological. This is of course not inconsistent with the observations discussed above than some lipid transporters are promiscuous-MsMmpL3 transports TMM and binds PI, PG and CL; NPC1 can transport CHOL as well as fatty acids and is also inhibited by TMM, as discussed above. So having NPC2 proteins bind ERGO as well as PG, PI is not entirely unexpected. We also note that there are several plants that lack NPC2. For example, potatoes, tomatoes as well as some grasses. Nevertheless, it appears that most plants use a NCR1/NPC2 dimer for lipid transport, and the NCR1 proteins have the same "active site" sequences and structures as found in fungi, apicomplexans and animals.

\section{Common structural features of lipid transporters and their substrates in bacteria, protozoa,}

yeasts, plants and animals? As discussed above, it has been found that there are large effects on sphingolipid transport in the plant $A$. thaliana on misexpression of the NCR1 gene ${ }^{16}$, and similar results have been reported with S. cerevisiae ${ }^{14}$ and $T$. gondil ${ }^{15}$. The major sphingolipids in plants 
are the so-called GIPCs, glycosylinositolphosphoceramides, ${ }^{48}$ phospholipids that contain 2 (eudicot) or 3 (monocot) sugar residues, ${ }^{49}$ and similar glycosylated sphingolipids are found in yeasts and fungi ${ }^{50}$ as well as in $T$. cruzi, but not in other trypanosomatids ${ }^{51}$

What is intriguing here is that there appears to be a pattern involving glycosylated lipid transport. Specifically, in the bacterium M. tuberculosis, trehalose monomycolate (TMM, 7) is transported by MmpL3. In S. cerevisiae, the apicomplexan T. gondii and in the plant A. thaliana, disruption of NCR1 function results in sphingolipid accumulation but does not affect sterol levels, and certainly in plants and fungi the major sphingolipids are GIPCs that have at least two sugars, and in T. gondii it has been shown that disruption of a TgNCR1 gene correlates with increased sphingolipid (GM1) accumulation. Both $\alpha$-MMPLs as well as the $\beta$-MMPLs are thus actually known to be involved in glycolipid or glycophospholipid trafficking, which supports the idea that in $T$. cruzi, the TcMMPL may play a role in GPI/GIPC transport, involved in this case in mucin biosynthesis. In $T$. gondii, there is no requirement for an $\alpha$-MMPL for sphingolipid transport while in $T$. cruzi, there is no need for a $\beta$-MMPL, for mucin biosynthesis.

It is also of interest to compare the domain structures in the $\alpha$ - and $\beta$-MMPLs, as well as the structures of known or proposed lipids that they bind or transport, as shown in Figure 7. Since different domains have different names in different proteins, for simplicity we use an A-E convention. The $\alpha$-domain proteins such as MsMmpL3 (PDB ID code 6OR2), SaFarE and TcMMPL contain an ABC domain structure in which there is an A domain consisting of $\sim 12 \mathrm{TM}$ helices together with extra-membranous $\mathrm{B}$ and $\mathrm{C}$ domains. The first half of each sequence is shown in cyan, the second in green, in Figures 7a-c. In the NPC1L1 cholesterol transporter, the ABC domains are again present but there is the additional D domain, shown in orange in Figure 7d (PDB ID code 6V3F). In the Solanum lysopersicum (tomato) homolog, the predicted structure is very 
similar, Figure 7e. In $T$. gondii NCR1 only $\sim 70 \%$ of the residues were predicted, the ABC domains shown in Figure 7f, and it appears that the $\mathrm{D}$ domain is just different to that in the other $\beta$-domain proteins. Similar results were obtained with PfNCR1. In earlier work, Coppens et al. ${ }^{15}$ found that while there were many sequence identities between the N-terminal residues in HsNPC1 and TgNCR1, there were 7 deletions and about $30 \%$ of the residues seen in HsNPC1 were missing in TgNCR1, making it difficult to predict the smaller D-domain structure (shown as an orange sphere in Figure 7f. TgNCR1 can restore sterol and GM1 trafficking when ectopically expressed in mammalian cells with impaired NPC1 activity ${ }^{15}$, but is primarily involved in controlling the intracellular levels of specific lipids, sphingolipids, in the parasite ${ }^{15}$. So, both cholesterol and sphingolipids can be transported but in the normal course of events, in the parasite, it appears that TgNCR1 is involved in sphingolipid transport, as also found in fungi.

In the NPC1/NPC2 structure (PDB ID code 6W5V) as well as the S. cerevisiae NCR1/NPC2 structure (PDB ID codes $6 \mathrm{R} 4 \mathrm{~L}+6 \mathrm{R} 4 \mathrm{~N}$, aligned to $6 \mathrm{~W} 5 \mathrm{~V}$ ) there is the addition of an $\mathrm{E}$ domain, Figures $7 \mathrm{~g}, \mathrm{~h}$, the NPC2 protein being shown in pink. In most plants, the same ABCDE domain structure is predicted, as shown for example in A. thaliana NCR1/NPC2, Figure 7i. Of interest here, BLAST searches of plant genomes did not return any hits when using the human NPC2 protein, but there were large numbers of hits when using the fungal NPC2. 

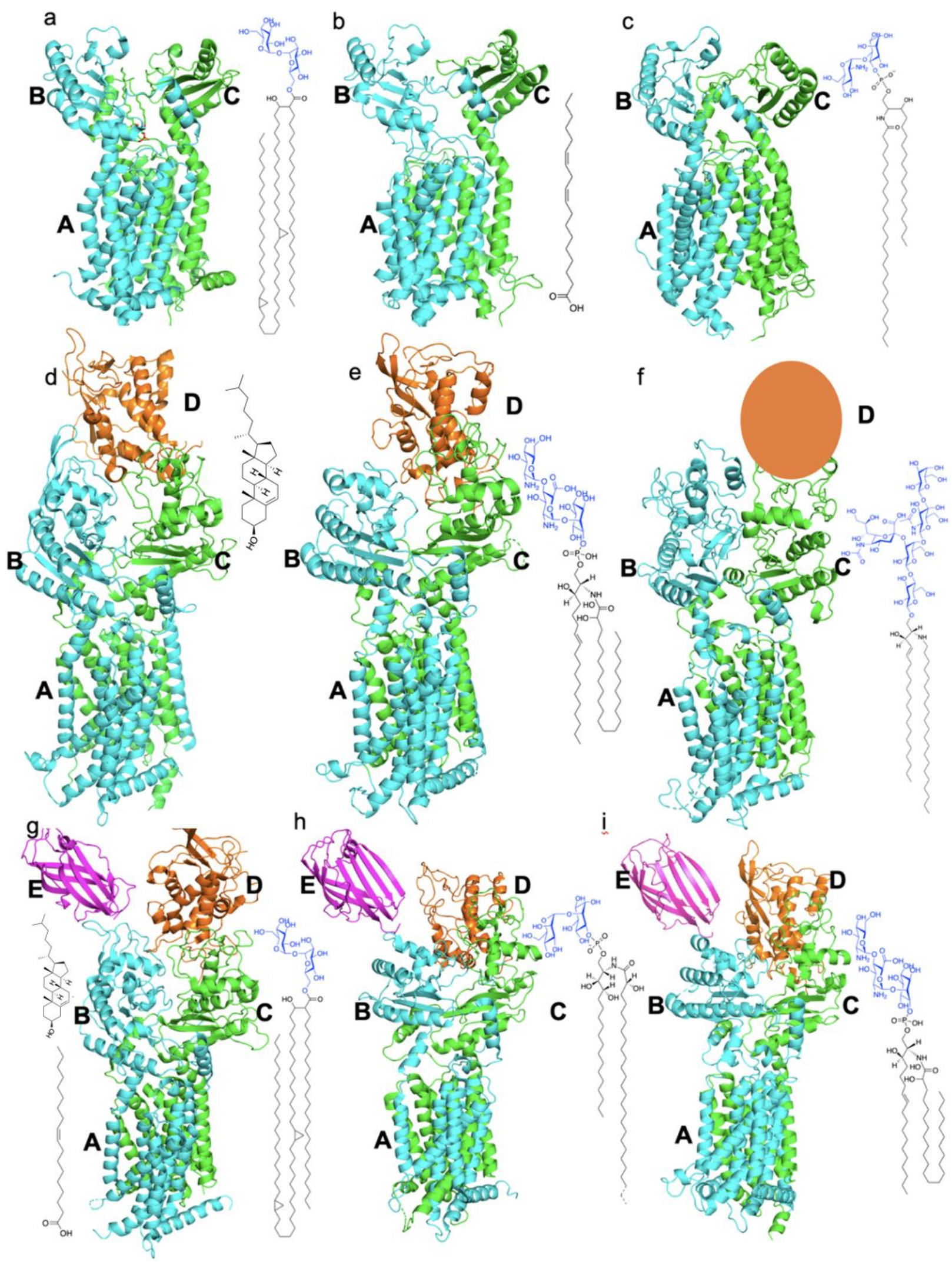
Figure 7. Domain structures in $\alpha$ - and $\beta$-MMPL proteins. a) MsMmpL3 (PDB ID code 6OR2). b) SaFarE Phyre2 prediction. c) TcMMPL Phyre2 prediction. d) Rattus norvegicus NPC1L1 (PDB ID code 6V3F). e) Solanum lycopersicum (tomato) Phyre2 prediction. f) TgNCR1 Phyre2 prediction. The D-domain was not modeled so is just shown as an orange sphere. g) HsNPC1/NPC2 (PDB ID code 6W5V). h) S. cerevisiae NCR1/NPC2 (PDB ID codes 6R4L+6R4N, aligned to 6W5V). i) A. thaliana NCR1/NPC2 Phyre2 prediction (aligned to 6W5V). The color codes are cyan and green represent the first and second halves of the $\alpha$-MMPL proteins (the $\mathrm{ABC}$ domains) that are also conserved in the larger proteins. Orange is the $\mathrm{N}$-terminal domain (D) in NPC1/NCR1/NCP1L1; and pink is NPC2 (E). Also shown are the chemical structures (from Figure 1a) of known or likely lipids that are transported or bind.

The E domain shows considerable plasticity with respect to what can bind, with CHOL, ERGO PG and PI all being reported. And in the plant A. thaliana, disruption of NPC1 results in sphingolipid accumulation, suggesting that sphingolipids may also bind to NPC2, as in fungi. Overall then, the basic NPC1/NPC2 domain structures are conserved in many organisms as are the $\mathrm{P} 1$ and $\mathrm{P} 2 \mathrm{H}^{+}$-transporter motifs, but what actually binds is very variable, with as noted above, the glycolipid TMM inhibiting NPC1 which can itself transport both cholesterol as well as oleic acid. We thus show in Figure 7 some of the diverse lipids that are known to be transported by or are known to bind to, these proteins, together with some proposals for other candidates. In many cases (MsMmpL3, TgNCR1, ScNCR1/NPC2, AtNCR1/NPC2), glycolipids (e.g. PI, TMM, GIPCs and GM1) are found to bind or accumulate on gene disruptions but it appears that only NPC1L1 and 
NPC1/NPC2 are actually involved in sterol (CHOL) transport. In T. cruzi, the role of TcMMPL is not known, but as noted above, GIPC (and GPI) transporters are required for mucin biosynthesis and are absent in other trypanosomatids, leading to the idea that TcMMPL may be a GIPC/GPI transporter.

In vivo activity of SQ109 against T. cruzi, T. brucei and L. donovani. Next, we investigated the effects of SQ109, in vivo, using mouse models of infection.

a
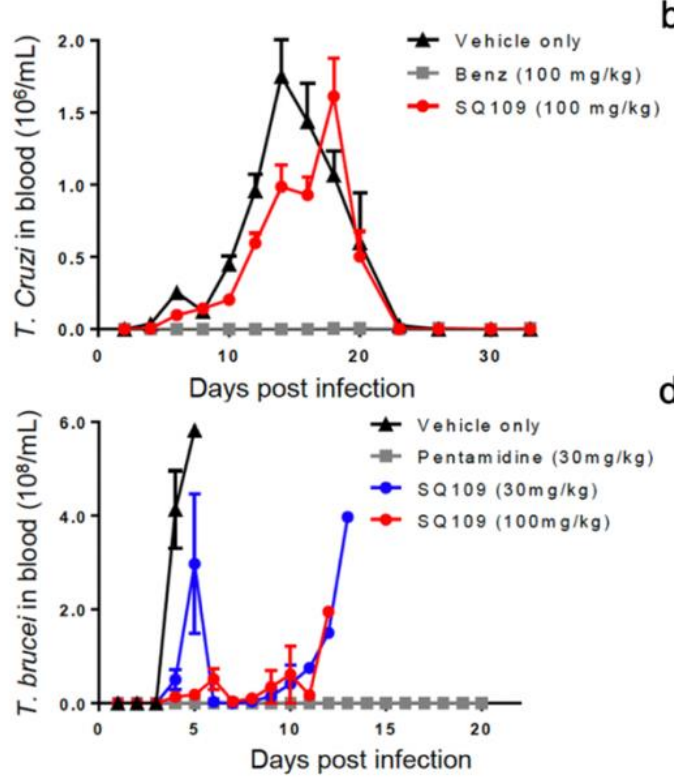

e

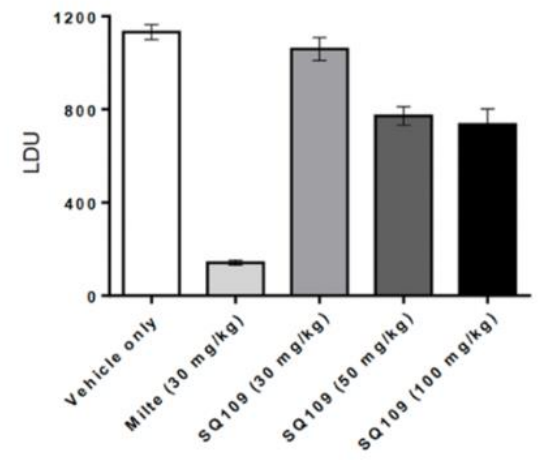

b
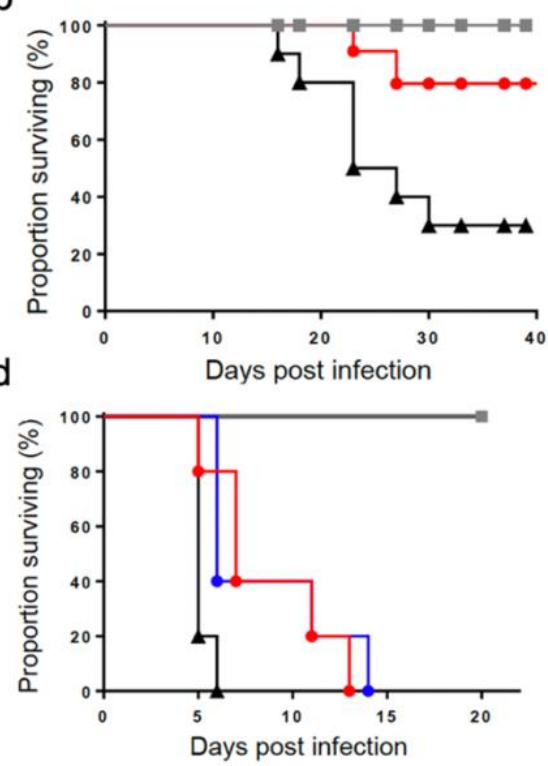

f

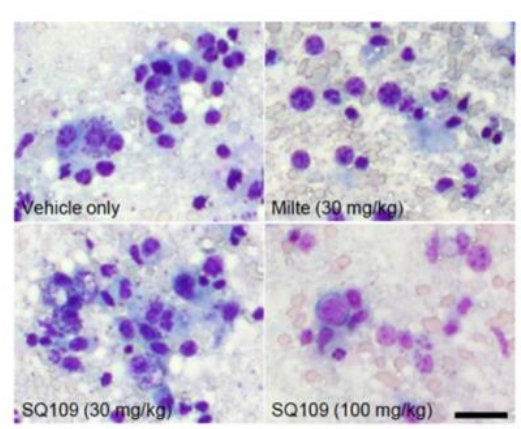

Figure 8. Efficacy of SQ109 against T. cruzi Dm28c, T. brucei and L. donovani Balb/c mouse models of infection. a) Number of $T$. cruzi parasites in bloodstream and b) survival of $T$. cruzi 
Dm28c infected Balb/cmice. Vehicle only [ $\mathbf{\Lambda}]$, benznidazole $(100 \mathrm{mg} / \mathrm{kg})[\mathbf{-}]$ or SQ109 (100 $\mathrm{mg} / \mathrm{kg})[\bullet]$ treated mice $(n=10)$. c) Number of $T$. brucei parasites in blood and d) mice survival. Vehicle only [ $\mathbf{\Delta}$ ], pentamidine $(30 \mathrm{mg} / \mathrm{kg})[\square]$, SQ109 $(30 \mathrm{mg} / \mathrm{kg})[\bullet]$ or SQ109 $(100 \mathrm{mg} / \mathrm{kg})[\bullet]$ $(n=5)$. e) Efficacy of tested drugs and controls in LDU (Leishman-Donovan unit) and f) Giemsastained images of Balb/c mice infected with $L$. donovani and treated with vehicle only, miltefosine $(30 \mathrm{mg} / \mathrm{kg})$ or SQ109 $(30,50$, or $100 \mathrm{mg} / \mathrm{kg})(n=5)$. Scale bar $=100 \mu \mathrm{m}$.

For the T. cruzi acute model, Balb/c mice were infected with bloodstream form T. cruzi Dm28c, and parasitemia as well as mouse survival was evaluated. The peak of parasitemia for the vehicle treated group was seen at 14 days post infection, and there was a $46.1 \%$ parasite reduction with the group treated with $100 \mathrm{mg} / \mathrm{kg}$ of SQ109 via oral gavage (q.d. for 2 weeks). The peak of parasitemia for the drug treated group was 18 days post infection, which represents a 4-day delay compared to that of the vehicle control (Figure 8a). The benznidazole treated group showed complete suppression of parasitemia during the whole experimental period. In terms of survival, $80 \%$ of the SQ109-treated mice survived, whereas the vehicle group showed only a $25 \%$ survival. Benznidazole protected all mice from death at least up to 40 days after infection (Figure 8b). The lack of more potent activity of SQ109 in vivo is likely to be due to the fact that SQ109 has a rather short half-life in animals, due to hepatic metabolism ${ }^{52}$, and that combination therapies may be required, as found for example, with amiodarone+itraconazole ${ }^{53}$ (based on earlier in vitro work with amiodarone+posaconaziole $\left.{ }^{41}\right)$.

For the T. brucei acute model, Balb/c mice were infected with the bloodstream form of the parasite, and parasitemia in blood as well as mice survival was monitored. SQ109 successfully suppressed the parasite load in the blood for few days after the termination of administration, but 
parasitemia rapidly increased soon after (Figure 8c). In the SQ109-treated group (30 mg/kg, once a day for 5 days via oral administration), the average survival was 8.6 days, which is 3.4 days greater than the vehicle control (Figure 8d). An increased dose of $100 \mathrm{mg} / \mathrm{kg}$ showed a similar, 3.6 average days of extended survival (Figure 8d). However, all mice had died by days 13-15 while all mice survived with pentamidine.

For the VL acute mouse model, L. donovani amastigotes isolated from infected hamster spleens were used to infect Balb/c mice by the retro-orbital route and treatments were given for 5 days starting from 5 days post infection. After 5 days of drug treatment, mice were sacrificed for the assessment of Leishman- Donovan units (LDU) from the collected livers. The parasite burden (in LDU) of the vehicle-treated group was $1132 \pm 73$, and those of SQ109-treated mice were $1060 \pm$ $110,772 \pm 89$ and $737 \pm 145$ for the 30,50 , and $100 \mathrm{mg} / \mathrm{kg}$ dosed groups, respectively, Figure 8e. There was a significant reduction of parasite burden with $50 \mathrm{mg} / \mathrm{kg}$ and $100 \mathrm{mg} / \mathrm{kg}$ dosing of SQ109 by $31.8 \%$ and $34.9 \%$, respectively, but even the best result was considerably lower than that with the miltefosine treatment which showed an LDU of $142 \pm 24$ (87.5\% parasite reduction), Figure 8e. As seen in the Giemsa stained liver impression smears, Figure 8f, amastigotes are still seen with SQ109-treated mice, whereas there is a dramatic decrease in parasites observed with the miltefosine treated group.

Toxoplasma gondii: In vitro and in vivo results with SQ109. The observation that SQ109 inhibits the growth of $P$. falciparum suggested the possibility that SQ109 might also inhibit the growth of another apicomplexan parasite, Toxoplasma gondii, the causative agent of toxoplasmosis. $T$. gondii, as with P. falciparum, does not synthesize either CHOL or ERGO and instead, as with $P$. falciparum, imports CHOL from its host cells, a process expected to require a CHOL transporter. 
There is no $\alpha$-MMPL protein found in the apicomplexans, so a possible candidate is a $\beta$-MMPL protein. Indeed, both host as well as parasite CHOL transport might be inhibited. We thus first determined whether SQ109 did actually inhibit the growth of $T$. gondii. Results are shown in Figure 9a in which we find an $\mathrm{IC}_{50}$ of $1.82 \mu \mathrm{M}$. This was a promising result so we next obtained 4 predicted TgN-R1 structures (e.g. Figure 7f) but unfortunately, only $\sim 70 \%$ of each sequence was predicted - the ABC domains - indicating some considerable structural differences (in the $\mathrm{N}$ terminal domain) to those found with the plant and animal proteins. Also of note is the observation by Coppens et al. that disruption of TgNCR1 resulted in accumulation of sphingolipids including GM1 and no effects on sterol levels, plus, mutant parasites were viable. It thus appears that unlike PfNCR1, TgNCR1 is not a good drug target. Nevertheless, since we did observe good in vitro activity, we moved on to investigate an in vivo model with Swiss Webster mice infected with a lethal dose of $T$. gondii tachyzoites, the fast-growing form of the parasite, since we did obtain good in vitro data.

Results are shown in Figure 9b and 9c. All untreated mice died 10 days post infection but there was a $~ 50-80 \%$ survival of the i.p. SQ109-treated mice, Figure 9b, and a 30-40\% survival at 40 days post-infection using oral gavage, Figure 9c. Thus, SQ109 kills T. gondii and has moderate activity in vivo. However, at day 42, we challenged the live animals with 100 T. gondii parasites, a control to verify that they were infected initially. The animals (4 out of 6) treated with 20 $\mathrm{mg} / \mathrm{kg} /$ day survived the challenge because the lower dose allowed the initial infection to become established and later recover and this allowed them develop immunity. However, for the ones treated with $50 \mathrm{mg} / \mathrm{kg} / \mathrm{day}$, the infection did not get established because the higher dose of SQ109 eliminated the parasites before the mice developed a robust immune response to re-infection and 
with the challenge at 42 days, these animals all died, a not uncommon observation in the development of drugs to treat Toxoplasma infections ${ }^{54,55}$.
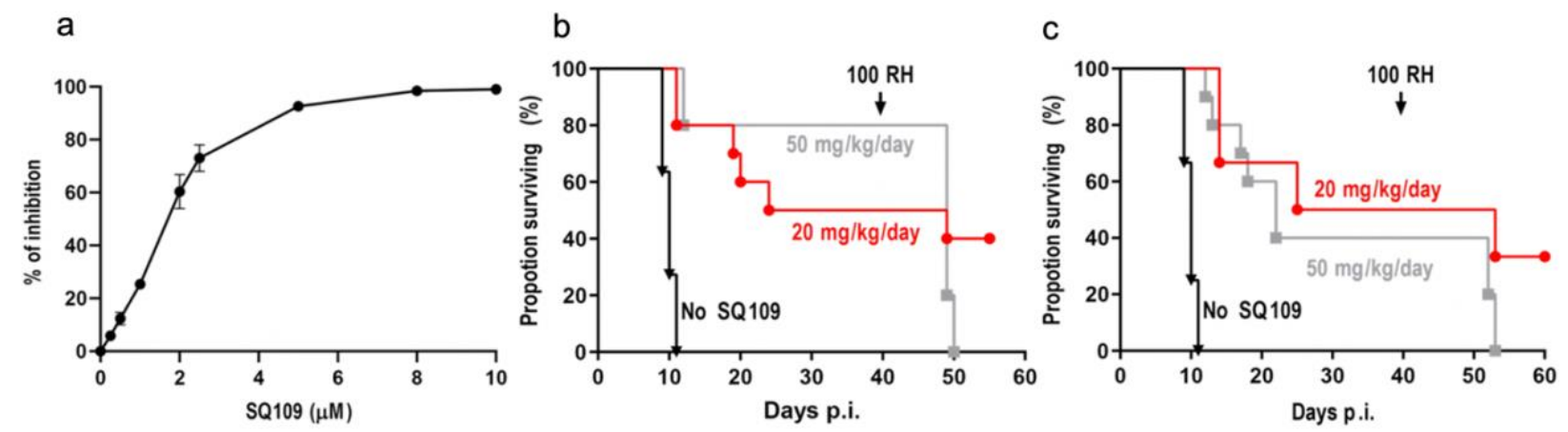

Figure 9. Effects of SQ109 on T. gondii cell growth inhibition and in vivo activity. a) T. gondii cell growth inhibition. IC50=1.82 $\mu \mathrm{M}$. b) $T$. gondii infected mice treated with SQ109 i.p. c) $T$. gondii infected mice treated with SQ109 by oral gavage.

\section{Conclusions}

The results we have discussed above are of interest for several reasons. First, stimulated by the observation that the TB drug candidate SQ109 that inhibits the trehalose monomycolate transporter MmpL3 also kills other pathogens, we find that there are MmpL3-like (MMPL) proteins in archaea, other bacteria, yeasts, protozoa, fungi, plants and animals. Based on sequence and structural data we define two classes: $\alpha$-MMPLs, lipid (e.g. fatty acid, TMM) transporters found in some bacteria and protozoa and $\beta$-MMPLs, sterol and sphingolipid transporters found in fungi, apicomplexans, plants and animals. Second, we find that in T. cruzi as well as in E. histolytica, important human pathogens, there are very similar proteins to MtMmpL3. These proteins are also present in 20 other protists but are not found in other trypanosomatids. Third, based on structure 
prediction algorithms, we show that the protein fold in TcMMPL and EhMMPL is the same as in the bacterial MsMmpL3 where there are two conserved DYxxF motifs that can bind to the ethylenediamine moiety in SQ109 (via electrostatic interactions with the conserved Asp), and hydrophobic interactions between the adamantane group and the "active site" Tyr and Phe residues. We call these the two pentad motifs, P1 and P2. Fourth, we propose that both the TcMMPL and EhMMPL proteins can act as GPI/GIPC transporters, involved in mucin biosynthesis in both species, based at least in part on the structural similarity between GPI and the PI that is known to bind to MsMmpL3. Fifth, we show that both $C$. difficile and S. aureus have similar MMPL proteins that in S. aureus, acts as a fatty acid efflux pump, FarE. Sixth, we show that MsMmpL3 has strong structural similarities to the transmembrane domain in three animal proteins: patched, dispatched and Niemann-Pick C1, as well as to the yeast NPC1 homolog and using these structures we find very similar proteins (and local, active site structures) in the apicomplexan parasites $P$. falciparum and $T$. gondii. All of these proteins we class as $\beta$-MMPLs. They have the same TM structure as the $\alpha$-MMPLs but have larger extra-membranous domains, involved in sterol or sphingolipid transport. They contain P1 and P2 pentad motifs that co-locate to the P1 and P2 motifs in the $\alpha$-MMPLs, but there are key residues changes, in particular, a Phe>His change in P2. Seventh, we show that there are two patches of highly conserved Ser/Thr motifs (typically containing a total of $5 \mathrm{Ser} / \mathrm{Thr}$ ) in the $\beta$-MMPls and that these are involved in hydrogen bonding to the catalytic Asp/Glu/His residues, and are involved in $\mathrm{H}^{+}$-transport. Eighth, we report the first in vivo results for SQ109 in T. cruzi, T. brucei, L. donovani and T. gondii, finding promising activity with $T$. cruzi.

Overall, our results indicate that there are basically two classes of MMPL proteins that have similar 3D folds and active site (TM) structures: $\alpha$-MMPLs that are involved in export processes 
such as TMM export, fatty acid efflux and most likely GPI/GIPC transport, and $\beta$-MMPL proteins (NPC1/NPC1L1/NCR1) in the apicomplexans, fungi, plants and animals that in most cases are involved in cholesterol or sphingolipid transport. The principal difference in the active site region-involved in $\mathrm{H}^{+}$-transport-between the $\alpha$-MMPL and $\beta$-MMPL proteins is the introduction of a protonatable active site His in the latter together with introduction of five Ser/His residues, which correlates with ligand (sterol, fatty acid and acriflavine) import rather than export. The results are also of interest since they indicate why the TB drug candidate SQ109 targets organisms other than $M$. tuberculosis and leads to the idea that both $\alpha$-MMPLs as well as $\beta$ MMPLs may be new drug targets for a diverse infectious diseases.

\section{Materials and methods}

Compounds. For T. brucei, pentamidine (Sigma) was dissolved in dimethyl sulfoxide (DMSO) and used as reference drug ${ }^{56}$. For T. cruzi assays, benznidazole (Carbosynth Limited) was prepared in DMSO and used as a reference drug ${ }^{57}$. For L. donovani assays, miltefosine (MedChemExpress) was prepared in water and used as a reference drug ${ }^{58}$. The final concentrations of DMSO did not exceed $0.6 \%$ of the total in vitro assay volume and $10 \%$ of the drug preparation used for in vivo administration, respectively, levels that are not toxic to the parasite, mammalian cells, and mice.

T. brucei in vivo model and drug efficacy test. Five-week-old female BALB/c mice were infected with T. b. brucei Lister 427 ( $4 \times 10^{4}$ cells) by intraperitoneal (i.p.) injection. The mice were divided into groups $(n=5)$, and drug treatment was performed for five consecutive days, starting from day 1 post-infection by administering $30 \mathrm{mg} / \mathrm{kg}$ of pentamidine, or $30 \mathrm{mg} / \mathrm{kg}, 100 \mathrm{mg} / \mathrm{kg}$ of SQ109, respectively. Drugs were freshly prepared each day. All drugs were administered once daily for 5 days via the per os route. Parasitemia was evaluated daily for 2 weeks by blood collection from 
the mouse tail vein, and survival was monitored for 1 month. Mice showing impaired health status and/or with a parasite load $>10^{8}$ cells per $\mathrm{mL}$ of blood, were euthanized.

T. cruzi in vivo model and drug efficacy test. BALB/c mice were infected with tissue culture trypomastigotes of $T$. cruzi $\operatorname{Dm} 28 \mathrm{c}\left(10^{7}\right.$ cells) by intraperitoneal (i.p.) injection. 5 days post infection, infected mice were sacrificed to collect blood stream form (BSF) T. cruzi. BALB/c mice were intraperitoneally infected with BSF T. cruzi Dm28c $\left(5 \times 10^{4}\right.$ cells $) .5$ days post infection collected $2^{\text {nd }}$-round BSF $\left(3 \times 10^{4}\right.$ cells $)$ were injected into BALB/c mice. Mice were divided into groups $(n=10)$, and orally treated with drugs for five consecutive days for 2 weeks, starting from day 2 post-infection, administering DPBS (Dulbecco's phosphate buffered saline), $100 \mathrm{mg} / \mathrm{kg}$ of benznidazole, or $100 \mathrm{mg} / \mathrm{kg}$ of SQ109, respectively. Parasitemia was evaluated every 2 days for 5 weeks by blood collection from the mouse tail vein, and survival was monitored for 2 months. Mice showing impaired health status were euthanized.

L. donovani in vivo model and drug efficacy test. BALB/c mice $(n=5)$ were injected with $2 \times$ $10^{7}$ hamster spleen-derived $L$. donovani amastigotes via the retro-orbital venous sinus route. From day 7 post-infection, groups of mice were treated using the vehicle only, miltefosine (30 mg/kg), or SQ109 (30, 50, $100 \mathrm{mg} / \mathrm{kg})$. On day 16 post-infection, all animals were euthanized and assessed microscopically using Giemsa-stained liver imprints. Parasite burdens were measured by counting (blinded to treatment) the number of amastigotes per 1000 cell nuclei and multiplying this number by the liver weight (mg; Leishman-Donovan Unit: LDU) ${ }^{59}$. The LDU values for the drug-treated samples were compared to those of the untreated samples.

T. gondii in vivo model and drug efficacy test. We used a parasite strain expressing red fluorescent protein (td-tomato) for in vitro drug testing ${ }^{54,55}$. Tachyzoites were maintained in human fibroblasts (hTert cells). Human fibroblasts were cultured in 96-well plates for $24 \mathrm{~h}$ prior 
to the addition of 4,000 fluorescent tachyzoites/well. Fluorescence values were measured for 3 to 4 days, and both excitation $(544 \mathrm{~nm})$ and emission $(590 \mathrm{~nm})$ were read from the bottom of the plates in a Molecular Devices plate reader. For in vivo drug testing, experiments were carried out using 20 fresh $T$. gondii tachyzoites of the RH strain to infect Webster mice. Drugs were dissolved in 10\% Kolliphor HS 15 and were inoculated intraperitoneally (i.p.). Treatment was initiated $6 \mathrm{~h}$ after infection and administered daily for 10 days. Surviving mice were challenged with 1,000 RH tachyzoites 42 days after infection

Ethics. Female BALB/c mice (5-6 weeks old; body weight 15-20 g) and male Golden Syrian hamsters (5-6 weeks old; body weight 60-80 g) were purchased from Orientbio Inc. (Seongnamsi, Rep. of Korea) and Central Laboratory Animal Inc. (Seoul, Rep. of Korea), respectively. All animal handling and experiments were performed in compliance with the guidelines and principles established by the Korean Animal Protection Law (http://animalrightskorea.org). All protocols for animal experiments were reviewed and approved by the Institutional Animal Care and Use Committee (IACUC, protocol \#IPK-19002 for acute T.brucei, \# IPK-17006-2 for T.cruzi and \# IPK-16003-3 for VL) of the Institute Pasteur Korea. Mouse experiments in Georgia followed a reviewed and approved protocol by the Institutional Animal Care and Use Committee (IACUC) and followed U.S. Government principles for the Utilization and Care of Vertebrate Animals, Animal Protocol A2018 02-021.

\section{ASSOCIATED CONTENT}

\section{Supporting Information.}

Supporting Information is available free of charge at http://pubs.acs.org.

Materials and Methods, Table S1, Figures S1-S5 


\section{Author Information}

\section{Corresponding Authors}

Joo Hwan No - Leishmania Research laboratory, Institut Pasteur Korea, Seongnam-si, Republic of Korea; http://orcid.org/0000-0002-3896-9808; E-mail: joohwan.no@ip-korea.org Eric Oldfield - Department of Chemistry, University of Illinois at Urbana-Champaign, Urbana, IL 61801, USA; http://orcid.org/0000-0002-0996-7352; Email: eoldfiel@illinois.edu

\section{Authors}

Satish R. Malwal - Department of Chemistry, University of Illinois at Urbana-Champaign, Urbana, IL 61801, USA; http://orcid.org/0000-0001-7606-1932

Kyung-Hwa Baek - Leishmania Research laboratory, Institut Pasteur Korea, Seongnam-si, Republic of Korea; http://orcid.org/ 0000-0001-6130-281X

Trong-Nhat Phan - Leishmania Research laboratory, Institut Pasteur Korea, Seongnam-si, Republic of Korea; http://orcid.org/0000-0002-4696-7490

Hyeryon Lee - Leishmania Research laboratory, Institute Pasteur Korea, Seongnam-si, Republic of Korea; http://orcid.org/0000-0002-8566-7503

Zhu-Hong Li - Center for Tropical and Emerging Global Diseases and Department of Cellular Biology, University of Georgia, Athens, GA 30602, USA; http://orcid.org/0000-0003-4940-0729

Silvia N.J. Moreno - Center for Tropical and Emerging Global Diseases and Department of Cellular Biology, University of Georgia, Athens, GA 30602, USA; http://orcid.org/0000-00022041-6295

\section{Author Contributions}


S.R.M. and K.H.B. contributed equally to this work.

\section{Notes}

The authors declare no competing financial interests.

\section{Acknowledgements}

We thank Isabelle Coppens, Julio Urbina and Roberto Docampo for helpful suggestions and Catherine Li for carrying out the $T$. gondii growth inhibition assay. This work was supported by the University of Illinois Foundation, a Harriet A. Harlin Professorship (to E.O), by a National Research Foundation of Korea (NRF) grant funded by the Korean government (MSIT, NRF2017M3A9G6068246 and 2020R1A2C1101104), and by the United States Public Health Service (National Institutes of Health grant 5R21AI147661 to S.N.J.M).

\section{References}

1. Roatt, B. M.; de Oliveira Cardoso, J. M.; De Brito, R. C. F.; Coura-Vital, W.; de Oliveira Aguiar-Soares, R. D.; Reis, A. B., Recent advances and new strategies on leishmaniasis treatment. Appl. Microbiol. Biotechnol. 2020, 104, 8965-8977.

2. Lakwo, T.; Oguttu, D.; Ukety, T.; Post, R.; Bakajika, D., Onchocerciasis elimination: progress and challenges. Res. Rep. Trop. Med. 2020, 11, 81-95.

3. Chen, P.; Gearhart, J.; Protopopova, M.; Einck, L.; Nacy, C. A., Synergistic interactions of SQ109, a new ethylene diamine, with front-line antitubercular drugs in vitro. J. Antimicrob. Chemother. 2006, 58, 332-337.

4. Boeree, M. J.; Heinrich, N.; Aarnoutse, R.; Diacon, A. H.; Dawson, R.; Rehal, S.; Kibiki, G. S.; Churchyard, G.; Sanne, I.; Ntinginya, N. E.; Minja, L. T.; Hunt, R. D.; Charalambous, 
S.; Hanekom, M.; Semvua, H. H.; Mpagama, S. G.; Manyama, C.; Mtafya, B.; Reither, K.; Wallis, R. S.; Venter, A.; Narunsky, K.; Mekota, A.; Henne, S.; Colbers, A.; van Balen, G. P.; Gillespie, S. H.; Phillips, P. P. J.; Hoelscher, M.; Consortium, P., High-dose rifampicin, moxifloxacin, and SQ109 for treating tuberculosis: a multi-arm, multi-stage randomised controlled trial. Lancet Infect. Dis. 2017, 17, 39-49.

5. Veiga-Santos, P.; Li, K.; Lameira, L.; de Carvalho, T. M.; Huang, G.; Galizzi, M.; Shang, N.; Li, Q.; Gonzalez-Pacanowska, D.; Hernandez-Rodriguez, V.; Benaim, G.; Guo, R. T.; Urbina, J. A.; Docampo, R.; de Souza, W.; Oldfield, E., SQ109, a new drug lead for Chagas disease. Antimicrob. Agents Chemother. 2015, 59, 1950-1961.

6. Li, K.; Wang, Y.; Yang, G.; Byun, S.; Rao, G.; Shoen, C.; Yang, H.; Gulati, A.; Crick, D. C.; Cynamon, M.; Huang, G.; Docampo, R.; No, J. H.; Oldfield, E., Oxa, thia, heterocycle, and carborane analogues of SQ109: bacterial and protozoal cell growth inhibitors. ACS Infect. Dis. 2015, $1,215-221$.

7. Gil, Z.; Martinez-Sotillo, N.; Pinto-Martinez, A.; Mejias, F.; Martinez, J. C.; Galindo, I.; Oldfield, E.; Benaim, G., SQ109 inhibits proliferation of Leishmania donovani by disruption of intracellular $\mathrm{Ca}(2+)$ homeostasis, collapsing the mitochondrial electrochemical potential $(\Delta \Psi(\mathrm{m}))$ and affecting acidocalcisomes. Parasitol. Res. 2020, 119, 649-657.

8. García-García, V.; Oldfield, E.; Benaim, G., Inhibition of Leishmania mexicana Growth by the Tuberculosis Drug SQ109. Antimicrob. Agents Chemother. 2016, 60, 6386-9.

9. Tahlan, K.; Wilson, R.; Kastrinsky, D. B.; Arora, K.; Nair, V.; Fischer, E.; Barnes, S. W.; Walker, J. R.; Alland, D.; Barry, C. E., 3rd; Boshoff, H. I., SQ109 targets MmpL3, a membrane 
transporter of trehalose monomycolate involved in mycolic acid donation to the cell wall core of Mycobacterium tuberculosis. Antimicrob. Agents Chemother. 2012, 56, 1797-1809.

10. Zhang, B.; Li, J.; Yang, X.; Wu, L.; Zhang, J.; Yang, Y.; Zhao, Y.; Zhang, L.; Cheng, X.; Liu, Z.; Jiang, B.; Jiang, H.; Guddat, L. W.; Yang, H.; Rao, Z., Crystal structures of membrane transporter MmpL3, an anti-Tb drug target. Cell 2019, 176, 636-648.

11. Sacksteder, K. A.; Protopopova, M.; Barry, C. E., 3rd; Andries, K.; Nacy, C. A., Discovery and development of SQ109: a new antitubercular drug with a novel mechanism of action. Future Microbiol. 2012, 7, 823-837.

12. Li, K.; Schurig-Briccio, L. A.; Feng, X.; Upadhyay, A.; Pujari, V.; Lechartier, B.; Fontes, F. L.; Yang, H.; Rao, G.; Zhu, W.; Gulati, A.; No, J. H.; Cintra, G.; Bogue, S.; Liu, Y. L.; Molohon, K.; Orlean, P.; Mitchell, D. A.; Freitas-Junior, L.; Ren, F.; Sun, H.; Jiang, T.; Li, Y.; Guo, R. T.; Cole, S. T.; Gennis, R. B.; Crick, D. C.; Oldfield, E., Multitarget drug discovery for tuberculosis and other infectious diseases. J. Med. Chem. 2014, 57, 3126-39.

13. Radhakrishnan, A.; Rohatgi, R.; Siebold, C., Cholesterol access in cellular membranes controls Hedgehog signaling. Nat. Chem. Biol. 2020, 16, 1303-1313.

14. Malathi, K.; Higaki, K.; Tinkelenberg, A. H.; Balderes, D. A.; Almanzar-Paramio, D.; Wilcox, L. J.; Erdeniz, N.; Redican, F.; Padamsee, M.; Liu, Y.; Khan, S.; Alcantara, F.; Carstea, E. D.; Morris, J. A.; Sturley, S. L., Mutagenesis of the putative sterol-sensing domain of yeast Niemann Pick C-related protein reveals a primordial role in subcellular sphingolipid distribution. J. Cell. Biol. 2004, 164, 547-56. 
15. Lige, B.; Romano, J. D.; Bandaru, V. V.; Ehrenman, K.; Levitskaya, J.; Sampels, V.; Haughey, N. J.; Coppens, I., Deficiency of a Niemann-Pick, type C1-related protein in toxoplasma is associated with multiple lipidoses and increased pathogenicity. PLoS Pathog. 2011, 7, e1002410.

16. Feldman, M. J.; Poirier, B. C.; Lange, B. M., Misexpression of the Niemann-Pick disease type C1 (NPC1)-like protein in Arabidopsis causes sphingolipid accumulation and reproductive defects. Planta 2015, 242, 921-33.

17. Long, T.; Qi, X.; Hassan, A.; Liang, Q.; De Brabander, J. K.; Li, X., Structural basis for itraconazole-mediated NPC1 inhibition. Nat. Commun. 2020, 11, 152.

18. Fineran, P.; Lloyd-Evans, E.; Lack, N. A.; Platt, N.; Davis, L. C.; Morgan, A. J.; Höglinger, D.; Tatituri, R. V. V.; Clark, S.; Williams, I. M.; Tynan, P.; Al Eisa, N.; Nazarova, E.; Williams, A.; Galione, A.; Ory, D. S.; Besra, G. S.; Russell, D. G.; Brenner, M. B.; Sim, E.; Platt, F. M., Pathogenic mycobacteria achieve cellular persistence by inhibiting the NiemannPick Type C disease cellular pathway. Wellcome Open Res. 2016, 1, 18.

19. Su, C. C.; Klenotic, P. A.; Bolla, J. R.; Purdy, G. E.; Robinson, C. V.; Yu, E. W., MmpL3 is a lipid transporter that binds trehalose monomycolate and phosphatidylethanolamine. Proc. Natl. Acad. Sci. U. S. A. 2019, 116, 11241-11246.

20. Kelley, L. A.; Mezulis, S.; Yates, C. M.; Wass, M. N.; Sternberg, M. J., The Phyre2 web portal for protein modeling, prediction and analysis. Nat Protoc 2015, 10, 845-858.

21. Altschul, S. F.; Gish, W.; Miller, W.; Myers, E. W.; Lipman, D. J., Basic local alignment search tool. J. Mol. Biol. 1990, 215, 403-410. 
22. Krissinel, E.; Henrick, K., Secondary-structure matching (SSM), a new tool for fast protein structure alignment in three dimensions. Acta Crystallogr.D Biol. Crystallogr. 2004, 60, 22562268.

23. Kumar, N.; Su, C. C.; Chou, T. H.; Radhakrishnan, A.; Delmar, J. A.; Rajashankar, K. R.; Yu, E. W., Crystal structures of the Burkholderia multivorans hopanoid transporter HpnN. Proc. Natl. Acad. Sci. U. S. A. 2017, 114, 6557-6562.

24. Winkler, M. B. L.; Kidmose, R. T.; Szomek, M.; Thaysen, K.; Rawson, S.; Muench, S. P.; Wüstner, D.; Pedersen, B. P., Structural insight into eukaryotic sterol transport through Niemann-Pick Type C proteins. Cell 2019, 179, 485-497.

25. Hayakawa, E. H.; Yamaguchi, K.; Mori, M.; Nardone, G., Real-time cholesterol sorting in Plasmodium falciparum-erythrocytes as revealed by 3D label-free imaging. Sci. Rep. 2020, 10, 2794.

26. Coppens, I.; Joiner, K. A., Host but not parasite cholesterol controls Toxoplasma cell entry by modulating organelle discharge. Mol. Biol. Cell 2003, 14, 3804-3820.

27. Sievers, F.; Wilm, A.; Dineen, D.; Gibson, T. J.; Karplus, K.; Li, W.; Lopez, R.; McWilliam, H.; Remmert, M.; Söding, J.; Thompson, J. D.; Higgins, D. G., Fast, scalable generation of high-quality protein multiple sequence alignments using Clustal Omega. Mol. Syst. Biol. 2011, 7, 539.

28. Alnaseri, H.; Arsic, B.; Schneider, J. E.; Kaiser, J. C.; Scinocca, Z. C.; Heinrichs, D. E.; McGavin, M. J., Inducible expression of a resistance-nodulation-division-type efflux pump in Staphylococcus aureus provides resistance to linoleic and arachidonic acids. J. Bacteriol. 2015, 197, 1893-905. 
29. Davies, J. P.; Chen, F. W.; Ioannou, Y. A., Transmembrane molecular pump activity of Niemann-Pick C1 protein. Science 2000, 290, 2295-2298.

30. Myers, B. R.; Neahring, L.; Zhang, Y.; Roberts, K. J.; Beachy, P. A., Rapid, direct activity assays for Smoothened reveal Hedgehog pathway regulation by membrane cholesterol and extracellular sodium. Proc. Natl. Acad. Sci. U. S. A. 2017, 114, E11141-E11150.

31. Petrov, K.; Wierbowski, B. M.; Liu, J.; Salic, A., Distinct cation gradients power cholesterol transport at different key points in the Hedgehog signaling pathway. Dev. Cell. 2020, $55,314-327$.

32. Bidet, M.; Tomico, A.; Martin, P.; Guizouarn, H.; Mollat, P.; Mus-Veteau, I., The Hedgehog receptor patched functions in multidrug transport and chemotherapy resistance. Mol. Cancer Res. 2012, 10, 1496-1508.

33. Qian, H.; Wu, X.; Du, X.; Yao, X.; Zhao, X.; Lee, J.; Yang, H.; Yan, N., Structural basis of low-ph-dependent lysosomal cholesterol egress by NPC1 and NPC2. Cell 2020, 182, 98111.

34. Wang, W.; Peng, D.; Baptista, R. P.; Li, Y.; Kissinger, J. C.; Tarleton, R. L., Strainspecific genome evolution in Trypanosoma cruzi, the agent of Chagas disease. PLoS Pathog. 2021, 17, e1009254.

35. Almeida, I. C.; Gazzinelli, R. T., Proinflammatory activity of glycosylphosphatidylinositol anchors derived from Trypanosoma cruzi: structural and functional analyses. J. Leukoc Biol. 2001, $70,467-477$. 
36. Cardoso, M. S.; Junqueira, C.; Trigueiro, R. C.; Shams-Eldin, H.; Macedo, C. S.; Araújo, P. R.; Gomes, D. A.; Martinelli, P. M.; Kimmel, J.; Stahl, P.; Niehus, S.; Schwarz, R. T.; Previato, J. O.; Mendonça-Previato, L.; Gazzinelli, R. T.; Teixeira, S. M., Identification and functional analysis of Trypanosoma cruzi genes that encode proteins of the glycosylphosphatidylinositol biosynthetic pathway. PLoS Negl. Trop. Dis. 2013, 7, e2369.

37. Jain, M.; Karan, D.; Batra, S. K.; Varshney, G. C., Mucins in protozoan parasites. Front. Biosci. 2001, 6, D1276-1283.

38. Vats, D.; Vishwakarma, R. A.; Bhattacharya, S.; Bhattacharya, A., Reduction of cell surface glycosylphosphatidylinositol conjugates in Entamoeba histolytica by antisense blocking of E. histolytica GlcNAc-phosphatidylinositol deacetylase expression: effect on cell proliferation, endocytosis, and adhesion to target cells. Infect Immun 2005, 73, 8381-92.

39. Bolaños, J.; Betanzos, A.; Javier-Reyna, R.; García-Rivera, G.; Huerta, M.; Pais-Morales, J.; González-Robles, A.; Rodríguez, M. A.; Schnoor, M.; Orozco, E., EhNPC1 and EhNPC2 proteins participate in trafficking of exogenous cholesterol in Entamoeba histolytica trophozoites: relevance for phagocytosis. PLoS Pathog. 2016, 12, e1006089.

40. Pereira, M. G.; Nakayasu, E. S.; Sant'Anna, C.; De Cicco, N. N.; Atella, G. C.; de Souza, W.; Almeida, I. C.; Cunha-e-Silva, N., Trypanosoma cruzi epimastigotes are able to store and mobilize high amounts of cholesterol in reservosome lipid inclusions. PLoS One 2011, 6, e22359.

41. Benaim, G.; Sanders, J. M.; Garcia-Marchán, Y.; Colina, C.; Lira, R.; Caldera, A. R.; Payares, G.; Sanoja, C.; Burgos, J. M.; Leon-Rossell, A.; Concepcion, J. L.; Schijman, A. G.; Levin, M.; Oldfield, E.; Urbina, J. A., Amiodarone has intrinsic anti-Trypanosoma cruzi activity and acts synergistically with posaconazole. J. Med. Chem. 2006, 49, 892-899. 
42. Pongratz, P.; Kurth, F.; Ngoma, G. M.; Basra, A.; Ramharter, M., In vitro activity of antifungal drugs against Plasmodium falciparum field isolates. Wien Klin Wochenschr 2011, 123, 26-30.

43. Martins-Duarte Edos, S.; de Souza, W.; Vommaro, R. C., Itraconazole affects Toxoplasma gondii endodyogeny. FEMS Microbiol. Lett. 2008, 282, 290-298.

44. Reader, J.; van der Watt, M. E.; Taylor, D.; Le Manach, C.; Mittal, N.; Ottilie, S.; Theron, A.; Moyo, P.; Erlank, E.; Nardini, L.; Venter, N.; Lauterbach, S.; Bezuidenhout, B.; Horatscheck, A.; van Heerden, A.; Spillman, N. J.; Cowell, A. N.; Connacher, J.; Opperman, D.; Orchard, L. M.; Llinás, M.; Istvan, E. S.; Goldberg, D. E.; Boyle, G. A.; Calvo, D.; Mancama, D.; Coetzer, T. L.; Winzeler, E. A.; Duffy, J.; Koekemoer, L. L.; Basarab, G.; Chibale, K.; Birkholtz, L.-M., Multistage and transmission-blocking targeted antimalarials discovered from the open-source MMV pandemic response box. Nature Commun. 2021, 12, 269.

45. Qi, X.; Schmiege, P.; Coutavas, E.; Li, X., Two Patched molecules engage distinct sites on Hedgehog yielding a signaling-competent complex. Science 2018, 362, eaas8843.

46. Khan, S. U.; Khan, M. U.; Virani, S. S.; Khan, M. S.; Khan, M. Z.; Rashid, M.; Kalra, A.; Alkhouli, M.; Blaha, M. J.; Blumenthal, R. S.; Michos, E. D., Efficacy and safety for the achievement of guideline-recommended lower low-density lipoprotein cholesterol levels: a systematic review and meta-analysis. Eur. J. Prev. Cardiol. 2020.

47. Record, E.; Moukha, S.; Asther, M., Characterization and expression of the cDNA encoding a new kind of phospholipid transfer protein, the phosphatidylglycerol/phosphatidylinositol transfer protein from Aspergillus oryzae: evidence of a 
putative membrane targeted phospholipid transfer protein in fungi. Biochim. Biophys. Acta 1999, 1444, 276-282.

48. Gronnier, J.; Germain, V.; Gouguet, P.; Cacas, J. L.; Mongrand, S., GIPC: glycosyl inositol phospho ceramides, the major sphingolipids on earth. Plant Signal Behav. 2016, 11, e1152438.

49. Lenarčič, T.; Albert, I.; Böhm, H.; Hodnik, V.; Pirc, K.; Zavec, A. B.; Podobnik, M.; Pahovnik, D.; Žagar, E.; Pruitt, R.; Greimel, P.; Yamaji-Hasegawa, A.; Kobayashi, T.; Zienkiewicz, A.; Gömann, J.; Mortimer, J. C.; Fang, L.; Mamode-Cassim, A.; Deleu, M.; Lins, L.; Oecking, C.; Feussner, I.; Mongrand, S.; Anderluh, G.; Nürnberger, T., Eudicot plant-specific sphingolipids determine host selectivity of microbial NLP cytolysins. Science 2017, 358, 14311434.

50. Guimaraes, L. L.; Toledo, M. S.; Ferreira, F. A. S.; Straus, A. H.; Takahashi, H. K., Structural diversity and biological significance of glycosphingolipids in pathogenic and opportunistic fungi. Front. Cell Infect. Microbiol. 2014, 4, 138.

51. De Lederkremer, R. M.; Agusti, R.; Docampo, R., Inositolphosphoceramide metabolism in Trypanosoma cruzi as compared with other trypanosomatids. J. Eukaryot. Microbiol. 2011, 58, 79-87.

52. Jia, L.; Noker, P. E.; Coward, L.; Gorman, G. S.; Protopopova, M.; Tomaszewski, J. E., Interspecies pharmacokinetics and in vitro metabolism of SQ109. Br. J. Pharmacol. 2006, 147, $476-485$.

53. Madigan, R.; Majoy, S.; Ritter, K.; Luis Concepción, J.; Márquez, M. E.; Silva, S. C.; Zao, C. L.; Pérez Alvarez, A.; Rodriguez-Morales, A. J.; Mogollón-Mendoza, A. C.; Estep, J. 
S.; Benaím, G.; Paniz-Mondolfi, A. E., Investigation of a combination of amiodarone and itraconazole for treatment of American trypanosomiasis (Chagas disease) in dogs. J. Am. Vet. Med. Assoc. 2019, 255, 317-329.

54. Li, Z. H.; Li, C.; Szajnman, S. H.; Rodriguez, J. B.; Moreno, S. N. J., Synergistic activity between statins and bisphosphonates against acute experimental toxoplasmosis. Antimicrob Agents Chemother 2017, 61, e02628-16.

55. Szajnman, S. H.; Galaka, T.; Li, Z. H.; Li, C.; Howell, N. M.; Chao, M. N.; Striepen, B.; Muralidharan, V.; Moreno, S. N.; Rodriguez, J. B., In Vitro and in vivo activities of sulfurcontaining linear bisphosphonates against apicomplexan parasites. Antimicrob. Agents Chemother. 2017, 61, e01590-16.

56. Yang, G.; Zhu, W.; Wang, Y.; Huang, G.; Byun, S. Y.; Choi, G.; Li, K.; Huang, Z.; Docampo, R.; Oldfield, E.; No, J. H., In vitro and in vivo activity of multitarget inhibitors against Trypanosoma brucei. ACS Infect. Dis. 2015, 1, 388-98.

57. Perez-Molina, J. A.; Perez-Ayala, A.; Moreno, S.; Fernandez-Gonzalez, M. C.; Zamora, J.; Lopez-Velez, R., Use of benznidazole to treat chronic Chagas' disease: a systematic review with a meta-analysis. J. Antimicrob. Chemoth. 2009, 64, 1139-1147.

58. Vermeersch, M.; da Luz, R. I.; Tote, K.; Timmermans, J. P.; Cos, P.; Maes, L., In vitro susceptibilities of Leishmania donovani promastigote and amastigote stages to antileishmanial reference drugs: practical relevance of stage-specific differences. Antimicrob Agents Chemother 2009, 53, 3855-3859. 
59. Phan, T. N.; Baek, K. H.; Lee, N.; Byun, S. Y.; Shum, D.; No, J. H., In vitro and in vivo activity of mTOR kinase and PI3K inhibitors against Leishmania donovani and Trypanosoma brucei. Molecules 2020, 25, 1980. 


\section{TOC graphic}

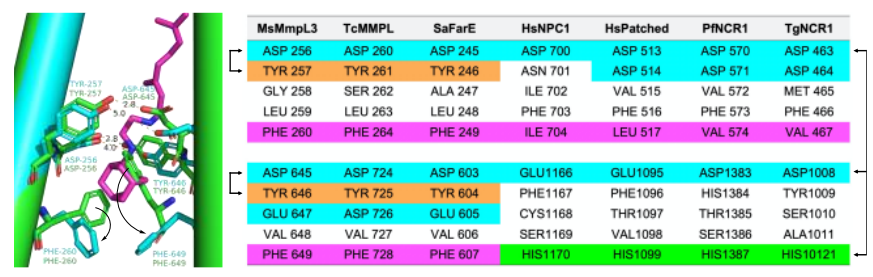

